\title{
Dynamic intuitionistic fuzzy multiattribute decision making based on evidential reasoning and MDIFWG operator
}

Liu, Y., Liu, J., \& Qin, Y. (2019). Dynamic intuitionistic fuzzy multiattribute decision making based on evidential reasoning and MDIFWG operator. Journal of Intelligent and Fuzzy Systems, 36(6), 5973-5987. https://doi.org/10.3233/JIFS-181777

Link to publication record in Ulster University Research Portal

Published in:

Journal of Intelligent and Fuzzy Systems

Publication Status:

Published (in print/issue): 11/06/2019

DOI:

10.3233/JIFS-181777

\section{Document Version}

Author Accepted version

\section{General rights}

Copyright for the publications made accessible via Ulster University's Research Portal is retained by the author(s) and / or other copyright owners and it is a condition of accessing these publications that users recognise and abide by the legal requirements associated with these rights.

\section{Take down policy}

The Research Portal is Ulster University's institutional repository that provides access to Ulster's research outputs. Every effort has been made to ensure that content in the Research Portal does not infringe any person's rights, or applicable UK laws. If you discover content in the Research Portal that you believe breaches copyright or violates any law, please contact pure-support@ulster.ac.uk. 


\title{
Dynamic Intuitionistic Fuzzy Multiattribute Decision Making Based on Evidential Reasoning and Modified Dynamic Intuitionistic Fuzzy Weighted Geometric Operator
}

\author{
Yi Liu \\ Data Recovery Key Laboratory of Sichuan Province, Neijiang Normal University, Neijiang 641000, Sichuan, P.R. China \\ School of Computing, Ulster University, Jordanstown Campus, Northern Ireland, UK \\ Jun Liu \\ School of Computing, Ulster University, Jordanstown Campus, Northern Ireland, UK
}

\begin{abstract}
The present work is focused on dynamic intuitionistic fuzzy multi-attribute decision making (DIF-MADM) problem, while dynamic means the decision-related information may be collected at different periods, a situation commonly happened in many of real world MADM problems. After the review and analysis of some drawbacks on the existing DIF-MADM methods, on the one hand, we propose a new DIF-MADM methods based on the evidential reasoning algorithm in order to address some of those limits; on the other hand, and a new dynamic intuitionistic fuzzy weighted geometric operator is introduced, named modified dynamic intuitionistic fuzzy weighted geometric (MDIFWG) operator, then a MDIFWG-based DIF-MADM method is also proposed to address some other limits of the existing methods. Some numerical examples are provided to illustrate the practicality and feasibility of the proposed two methods through, the comparative analysis with the existing DIF-MADM methods, along with some sensitivity analyses also carried out to analyse the distinct features of the proposed methods.
\end{abstract}

Keywords: Dynamic intuitionistic fuzzy multi-attribute decision making (DIF-MADM), Modified dynamic intuitionistic fuzzy weighted geometric (MDIFWG) operator, Evidential reasoning algorithm

\section{Introduction}

As an important extension of fuzzy set, Intuitionistic Fuzzy Set (IFS) [1, 2, 3] is characterized by three parameters at the same time, namely, a membership degree, a nonmembership degree and an indeterminacy degree are adopted at the same time. Therefore, IFS is considered to be more appropriate to represent and deal with imprecise, uncertain and vague information in some decision making problems. In last few years, some fuzzy multi-attribute decision making

Email addresses: liuyiyl@126.com, y.liu2@ulster.ac.uk (Yi Liu), j.liu@ulster.ac.uk (Jun Liu) 
methods based on IFS have been proposed, e.g., [5, 7, 10, 11, 16, 21, 30, 31, 32, 33, 36, 37, 42, $45,46]$, among others. All these studies are focused on the decision making problems where all the decision-related information are provided at the same period, however, those information 10 are usually collected at different periods in many real decision problems. To handle this type of situation, Xu and Yager [39] investigated dynamic intuitionistic fuzzy multi-attribute decision making (DIF-MADM) problems where all the attribute values are expressed as intuitionistic fuzzy numbers (IFNs) collected at different periods.

Regardless of the MADM problem based on IFS or DIF-MADM problem, aggregation of intuitionistic fuzzy information is always one of key research issues. Accordingly, many aggregation operators have been introduced under intuitionistic fuzzy environment and applied to different MADM problems, e.g., as far as IFS is concerned, intuitionistic fuzzy weighted averaging (IFWA) operator [34], intuitionistic fuzzy ordered weighted averaging (IFOWA) operators [34], intuitionistic fuzzy hybrid aggregation (IFHA) operator [40], intuitionistic fuzzy weighted 20 geometric (IFWG) operator [28, 38], intuitionistic fuzzy ordered weighted geometric (IFOWG) operators [38], intuitionistic fuzzy hybrid geometric (IFHA) operators [38] and other induced aggregation operators [17, 20, 29, 35, 43, 44]. In addition, different aggregation operators have been also introduced and applied into different DIF-MADM methods [4, 8, 12, 18, 19, 25, 27, 52], e.g., dynamic intuitionistic fuzzy weighted averaging (DIFWA) operator [39], uncertain dy-

25 namic intuitionistic fuzzy weighted averaging (UDIFWA) operator [39], dynamic intuitionistic fuzzy weighted geometric (DIFWG) operator [26, 32, 41], uncertain dynamic intuitionistic fuzzy weighted geometric (UDIFWG) operators [26, 32, 41], dynamic intuitionistic fuzzy weighted averaging Einstein (DIFWA ${ }^{\epsilon}$ ) operator and dynamic intuitionistic fuzzy weighted geometric Einstein $\left(\mathrm{DIFWG}^{\epsilon}\right)$ operator [15].

30 Although different aggregation operators have been introduced, they still cannot help to overcome the drawback of some existing DIF-MADM methods which result in unreasonable preference orders of alternatives in some decision situations [15, 26, 32, 39]. Motivated by this limitation in some existing DIF-MADM methods, this paper aims at proposing new DIF-MADM strategy and new aggregation operators and evaluates their feasibility and performance compared with the existing work.

In order to improve the DIF-MADM method, we proposed to use new strategy based on evidential reasoning (ER) methodology. On the basis of Dempster-Shafer Theory [13, 14], Yang and $\mathrm{Xu}[47,48]$ proposed an ER algorithm for MADA under uncertainty. Since then, ER methodology/algorithms have been successfully used in different decision making problems

$40 \quad[9,22,23,24,49,50,51,53]$. Specially, Yang et al. [51] presented an ER approach for MADA under both probabilistic and fuzzy uncertainties. Chen et al [10] took the advantage of the ER methodology and the representation capability of IFSs to propose a new fuzzy MADM method based on the ER methodology. Chen et al. [11] also proposed a new method for fuzzy MADM based on the transformation techniques between IFN and rightangled triangular fuzzy numbers

45 along with a new intuitionistic fuzzy geometric averaging operators of IFNs. The ER methodology has shown its potential capability in MADM and the likability to be incorporated with the DIF-MADM method, this is one of main focus of the present work.

Now that aggregation operators plays the key role in DIF-MADM method, in order to overcome the drawbacks of some existing DIF-MADM methods, the second focus of the present

50 work is on introducing and evaluating the new aggregation operators. Accordingly, a new dynamic intuitionistic fuzzy weighted geometric aggregation operators (MDIFWG) is proposed along with the corresponding DIF-MADM method. The remaining of the paper is organized as follows: Section 2 includes preliminary concepts and definitions relevant, such as IFS and 
intuitionistic fuzzy variable, score function, and evidential reasoning algorithm. In Section 3,

55 we provide the formal description of DIF-MADM problems and review and analyse some drawbacks of existing DIF-MADM methods. In Section 4, a new DIF-MADM methods based on the ER algorithm is proposed first (denoted as Method I) and then a new DIFWG operator named MDIFWG operator introduced along with the MDIFWG-based DIF-MADM method (denoted as Method II). In Section 5 focuses on the evaluation of the feasibility and validity of the proposed

60 DIF-MADM methods through some numerical examples and comparative analysis with some existing DIF-MADM method, along with some sensitivity analysis. This paper is concluded in Section 6.

\section{Preliminaries}

In this section, firstly some basic concepts related to intuitionistic fuzzy set and dynamic 65 intuitionistic fuzzy set are reviewed, along with an overview of the evidential reasoning algorithm $[47,48,50]$, which are the basis of the present work.

\subsection{Intuitionistic fuzzy set and intuitionistic fuzzy variable}

Definition 1. [1] Let $X=\left\{x_{1}, x_{2}, \cdots, x_{n}\right\}$ be a finite universe of discourse, an intuitionistic fuzzy set (IFS) $A$ in $X$ characterized by a membership function $\mu_{A}: X \rightarrow[0,1]$ and a non-membership function $v_{A}: X \rightarrow[0,1]$, which satisfies the condition $0 \leq \mu_{A}(x)+v_{A}(x) \leq 1$. An IFS A can be expressed as

$$
A=\left\{\left\langle x,\left(\mu_{A}(x), v_{A}(x)\right)\right\rangle \mid x \in X\right\} .
$$

$\pi_{A}(x)=1-\mu_{A}(x)-v_{A}(x)$ is called the degree of indeterminacy, $\pi_{A}(x)$ represents the degree of hesitance of $x$ to $A$ and is also called intuitionistic index. For convenience, called $\left(\mu_{A}(x), v_{A}(x)\right)$

70 is an intuitionistic fuzzy number (IFN) and denoted by $\left(\mu_{A}, v_{A}\right)$.

For an IFS $A$ on the universe $X, A$ will be reduced to a fuzzy set under the condition that intuitionistic index $\pi_{A}(x)=0$ for any $x \in X$.

Refer to [41], the intuitionistic fuzzy number $\left(v_{A}\left(x_{i}\right), \mu_{A}\left(x_{i}\right)\right)$ is the complement of a intuitionistic fuzzy number $\left(\mu_{A}\left(x_{i}\right), v_{A}\left(x_{i}\right)\right)$, denoted as $\left(\mu_{A}\left(x_{i}\right), v_{A}\left(x_{i}\right)\right)^{C}=\left(v_{A}\left(x_{i}\right), \mu_{A}\left(x_{i}\right)\right)$.

In MADM problem, aggregation operator plays an important role in combining relevant information from multiple sources. Xu and Yager [41] developed some aggregation operators to aggregate IF information. However, these operators can only be used to deal with time independent arguments. If time is taken into account, for example, the argument information may be collected at different periods, then these aggregation operators will not work effectively. Ac-

80 cordingly, Xu and Yager [39] proposed the concept of intuitionistic fuzzy variables, as shown below:

Definition 2. [39] Let $t$ be a time variable, then $\alpha(t)=\left(\mu_{\alpha(t)}, v_{\alpha(t)}\right)$ is called an intuitionistic fuzzy variable, where $\mu_{\alpha(t)} \in[0,1], v_{\alpha(t)} \in[0,1]$ and $\mu_{\alpha(t)}+v_{\alpha(t)} \in[0,1]$.

For an intuitionistic fuzzy variable $\alpha(t)$, if $t=t_{1}, t_{2}, \cdots, t_{k}$, then $\alpha_{t_{1}}, \cdots, \alpha_{t_{k}}$ indicate $k$ IFNs 85 collected at $p$ different periods. 


\subsection{Score function of decision-making problem}

Given a finite set of alternatives, an intuitionistic fuzzy MADM problem is a kind of problem in which the evaluation of each alternative with respect to a set of attributes is expressed by IFNs, and the most desirable alternative is selected based on the degree of suitability to which each al-

90 ternative satisfies the decision-makers requirements. However, the size relations or the inclusion relations does not exist in IFS under ambient conditions, some comparison technologies of IFNs have been developed to determine the order relations of IFNs. Score function, an important tool to evaluate IFNs in order to obtain the best alternative in decision making problem, is needed to convert IFNs into real numbers in order to become easier to compare with each other.

95 In the intuitionistic fuzzy MADM problem, as far as the score function is concerned, an effective score function has the following properties [29]: (1) the degree of membership, nonmembership and indeterminacy (hesitation) of IFS should be considered; (2) it should have higher precision; and (3) it should also have stronger selection ability.

Wang [29] analysed limitations of existing score functions for IFS, an effective score function is given based on the cross entropy of membership degree from the non-membership degree, it is used to determine the absolute value of influence difference that the membership degree and the non-membership degree responded to the hesitation degree. The cross-entropy [29] of the degree of membership from the non-membership based on IFS is defined as follows.

Definition 3. [29] Let $\alpha=(\mu, v)$ be an IFN of an IFS, the cross-entropy of the degree of membership $\mu$ from the degree of no-membership $v$ is called cross-entropy based on IFS, which measures the divergence between $\mu$ and $v$ :

$$
H(\alpha)=H(\mu, v)= \begin{cases}\log _{2} \frac{2}{2-v}, & \mu=0 \\ \log _{2} \frac{2}{1+v}, & \mu=1 \\ \mu \log _{2} \frac{2 \mu}{(\mu+v)}+(1-\mu) \log _{2} \frac{2(1-\mu)}{2-(\mu+v)}, & 0<\mu<1\end{cases}
$$

From Definition 3, it is obvious that $H(\mu, v) \neq H(v, \mu)$, that is, $H(\mu, v)$ is not symmetric. Therefore, Definition 3 should be modified as:

$$
H_{M}(\alpha)=\frac{H(\alpha)+H\left(\alpha^{C}\right)}{2}
$$

Theorem 1. [29] Let $\alpha=(\mu, v)$ be an IFN, then $H_{M}(\alpha)$ satisfies the following properties:

(1) $H_{M}(\alpha) \in[0,1]$;

(2) $H_{M}(\alpha)=H_{M}\left(\alpha^{C}\right)$;

(3) If $\alpha=(1,0)$ or $\alpha=(0,1)$, then $H_{M}(\alpha)=1$;

(4) If $\alpha=\alpha^{C}$, then $H_{M}(\alpha)=0$.

Entropy is very important for measuring uncertain information. As far as the cross-entropy defined in Eq. (2) is concerned, for a given IFN $\alpha=(\mu, v)$, if $H_{M}(\alpha)=0$, then the divergence between $\mu$ and $v$ responding to the degree of hesitation $\pi_{i}$ is the smallest; if $h_{M}(\alpha)=1$, then the divergence between $\mu$ and $v$ responding to the degree of hesitation $\pi_{i}$ is the largest.

In order to determine the best alternative in decision making problem, an effective score function is defined as follows to measure the degree of suitability to which the alternative satisfies the DM's requirement. 
Definition 4. Let $\alpha=(\mu, v)$ be an IFN. The new score function of $\alpha$ is defined as

$$
S(\alpha)= \begin{cases}\mu-v+H_{M}(\alpha) \pi & \mu>v \\ \mu-v-H_{M}(\alpha) \pi & \mu<v \\ 0^{*} & \mu=v\end{cases}
$$

where $\pi=1-\mu-v$ and $0^{*}$ means that $S$ is close to 0 .

For an IFN $\alpha=(\mu, v)$, the value of unknown degree $\pi=1-\mu-v$ is moderate under the condition $\mu=v$. As $\pi$ denotes degree of indeterminacy, hence the degree of accuracy of IFN $\alpha$ will change with $\pi$ change and indeterminacy of $\pi$ almost have little influence on score value of $\alpha$, so the value is close to 0 rather than equal to 0 . Only if $\pi=0$, i.e. $\mu=v=0.5$, the value of score equal to 0 , that is, the degree of indeterminacy is the smallest and the value of accuracy is the largest. For example, there are two alternatives: $\alpha_{1}=(0.5,0.5)$ and $\alpha_{2}=(0.3,0.3)$, it is obvious that $\pi_{1}<\pi_{2}$. Therefore, $S\left(\alpha_{2}\right)<S\left(\alpha_{1}\right)=0$, it follows that the alternative $\alpha_{1}$ is better than the alternative $\alpha_{2}$.

Theorem 2. Let $\alpha=(\mu, v)$ be an IFN. Then $S(\alpha)$ satisfies the following properties:

(1) $S(\alpha) \in[-1,1]$;

(2) $S(\alpha)=1$ if and only if $\alpha=(1,0)$;

(3) $S(\alpha)=-1$ if and only if $\alpha=(0,1)$;

(4) If $S(\alpha)=0$ if and only if $\alpha=(0.5,0.5)$.

For any two IFNs $\alpha_{1}, \alpha_{2}$,

(1) if $S\left(\alpha_{1}\right)<S\left(\alpha_{2}\right)$, then $\alpha_{1}<\alpha_{2}$;

(2) if $S\left(\alpha_{1}\right)>S\left(\alpha_{2}\right)$, then $\alpha_{1}>\alpha_{2}$;

(3) if $S\left(\alpha_{1}\right)>S\left(\alpha_{2}\right)$, then $\alpha_{1} \sim \alpha_{2}$.

Example 1. Let $\alpha_{1}=(0.52,0.2), \alpha_{2}=(0.7,0.3), \alpha_{3}=(0.12,0.68)$ be three IFNs. By Eq. (2), we have

$$
H_{M}\left(\alpha_{1}\right)=0.1519, H_{M}\left(\alpha_{2}\right)=0.1959, H_{M}\left(\alpha_{3}\right)=0.0841
$$

and so

$$
S\left(\alpha_{1}\right)=0.3625, S\left(\alpha_{2}\right)=0.4, S\left(\alpha_{3}\right)=-0.5432 \text {. }
$$

Therefore $\alpha_{3} \prec \alpha_{1} \prec \alpha_{2}$.

\subsection{Evidential reasoning algorithm for MADM}

In this subsection, we review the ER algorithm for MADM under uncertain environment $[50,49,51]$. Let $X=\left\{x_{1}, x_{2}, \cdots, x_{m}\right\}$ be a set of alternatives and $A=\left\{a_{1}, a_{2}, \cdots, a_{p}\right\}$ be a set of attributes. Assume that there are $N$ evaluation grades $\theta_{1}, \theta_{2}, \cdots, \theta_{N}$ for assessing the attributes of alternatives and denoted by $\Theta=\left\{\theta_{1}, \theta_{2}, \cdots, \theta_{N}\right\}, w_{i}$ refer to the weight of attribute $a_{i}(i=$

$1451,2, \cdots, p)$, respectively, with $w_{i} \in[0,1]$ and $\sum_{i=1}^{n} w_{i}=1$. Let $S\left(a_{i}\left(x_{j}\right)\right)$ denote the evaluation value of attribute $a_{i}$ of alternative $x_{j}$ and be defined as follows:

$$
S\left(a_{i}\left(x_{j}\right)\right)=\left\{\left(\theta_{n}, \beta_{n, i}\right)\left(x_{j}\right), n=1,2, \cdots, N\right\}
$$


where $i=1,2, \cdots, p$ and $j=1,2, \cdots, m$.

The assessments of the attributes of the alternatives are represented by a decision matrix $D=\left(S\left(a_{i}\left(x_{j}\right)\right)\right)_{p \times m}$. Now we aggregate the assessment values of attributes for all alternatives. According to Eq. (4), the belief of degree $\beta_{\theta_{n}, i}\left(x_{j}\right)$ regarding to the $i$ th attribute $a_{i}$ of alternative $x_{j}$ can be transformed into bps $m_{\theta_{n}, i}\left(x_{j}\right)$ as follows:

$$
\begin{aligned}
& m_{n, i}\left(x_{j}\right)=w_{i} \beta_{n, i}\left(x_{j}\right) ; \\
& m_{\Theta, i}\left(x_{j}\right)=1-\sum_{n=1}^{N} m_{\theta_{n}, i}\left(x_{j}\right)=1-w_{i} \sum_{n=1}^{N} \beta_{\theta_{n}, i}\left(x_{j}\right),
\end{aligned}
$$

where $n=1,2, \cdots, N, i=1,2, \cdots, p$ and $j=1,2, \cdots, m$.

Below is the results aggregating the criteria (or attribute) by combining the basic probability masses generated above, where $m_{n, I(1)}\left(x_{j}\right)=m_{n, 1}\left(x_{j}\right), m_{\Theta, I(1)}\left(x_{j}\right)=m_{\Theta, 1}\left(x_{j}\right)$,

$$
\begin{array}{ll}
\left\{\theta_{n}\right\}: & m_{n, I(i)}\left(x_{j}\right)=K\left[m_{n, I(i-1)}\left(x_{j}\right) m_{n, i}\left(x_{j}\right)+m_{n, I(i-1)}\left(x_{j}\right) m_{\Theta, i}\left(x_{j}\right)+m_{\Theta, I(i-1)}\left(x_{j}\right) m_{n, i}\left(x_{j}\right)\right] \\
\{\Theta\}: & m_{\Theta, I(i)}\left(x_{j}\right)=K\left[m_{\Theta, I(i-1)}\left(x_{j}\right) m_{\Theta, i}\left(x_{j}\right),\right. \\
& K=1-\sum_{r=1}^{N} \sum_{t=1, t \neq r}^{N} m_{m_{r}, I(i-1)}\left(x_{j}\right) m_{t, i}\left(x_{j}\right) \\
\left\{\theta_{n}\right\}: & \beta_{n}\left(x_{j}\right)=\frac{m_{n, I(p)}\left(x_{j}\right)}{1-m_{\Theta, I(p)}\left(x_{j}\right)} .
\end{array}
$$

From Eq. (6), we can obtain another equivalent form:

$$
\beta_{n}\left(x_{j}\right)=\frac{\left(1-\beta_{\Theta}\left(x_{j}\right)\right) m_{n, I(p)}\left(x_{j}\right)}{1-m_{\Theta, I(p)}\left(x_{j}\right)},
$$

where $\beta_{\Theta}\left(x_{j}\right)=\sum_{i=1}^{p} w_{i}\left(1-\sum_{n=1}^{N} \beta_{n, i}\left(x_{j}\right)\right)$.

\section{Analysis of the existing DIF-MADM methods}

In this section, we will review the formal representation of the typical DIF-MADM problem, and analyse their drawbacks, then in Section 4, we will introduce methods in order to overcome those drawbacks.

\subsection{Formal representation of DIF-MADM}

In general, MADM has always been used to find the most desirable one from a finite set of alternatives with respect to the predefined attributes. DIF-MADM methods aim at handling the MADM problems under dynamic intuitionistic fuzzy environment, especially on MADM problems with the subjective information and the attitudinal character of the decision makers. A DIF-MAGDM problem can be formally described as follows:

(1) $X=\left\{x_{1}, x_{2}, \cdots, x_{m}\right\}$ a set of $m$ alternatives;

(2) $A=\left\{a_{1}, a_{2}, \cdots, a_{n}\right\}$ the set of $n$ attributes whose weight vector is $w=\left(w_{1}, \cdots, w_{n}\right)$ with $w_{i}>0$ and $\sum_{i=1}^{n} w_{i}=1$;

(3) There are $p$ periods $P=\left\{t_{1}, t_{2}, \cdots, t_{p}\right\}$, whose weight vector is $\omega(t)=\left(\omega\left(t_{1}\right), \cdots, \omega\left(t_{p}\right)\right)$ with $\omega\left(t_{k}\right)>0(k=1,2, \cdots, p)$ and $\sum_{k=1}^{p} \omega\left(t_{k}\right)=1$. 
(4) The decision makers provide the attribute values of alternative $x_{i} \in X(i=1,2, \cdots, m)$ with respect to attribute $a_{j}(j=1,2, \cdots, n)$ at period $t_{k}(k=1,2, \cdots, p)$ and construct the intuitionistic fuzzy decision making matric

$$
D_{t_{k}}=\left(\alpha_{i j, t_{k}}\right)_{m \times n}=\left(\begin{array}{cccc}
\left(\mu_{11, t_{k}}, v_{11, t_{k}}\right) & \left(\mu_{12, t_{k}}, v_{12, t_{k}}\right) & \cdots & \left(\mu_{1 n, t_{k}}, v_{1 n, t_{k}}\right) \\
\left(\mu_{21, t_{k}}, v_{21, t_{k}}\right. & \left(\mu_{22, t_{k}}, v_{22, t_{k}}\right) & \cdots & \left(\mu_{2 n, t_{k}}, v_{2 n, t_{k}}\right) \\
\vdots & \vdots & \vdots & \vdots \\
\left(\mu_{m 1, t_{k}}, v_{m 1, t_{k}}\right) & \left(\mu_{m 2, t_{k}}, v_{m 2, t_{k}}\right) & \cdots & \left(\mu_{m n, t_{k}}, v_{m n, t_{k}}\right)
\end{array}\right)
$$

where $\left(\mu_{i j, t_{k}}, v_{i j, t_{k}}\right)$ is an IFN, $\mu_{i j, t_{k}}$ is the degree that alternative $x_{i}$ should satisfy the attribute $a_{j}$ at period $t_{k}, v_{i j, t_{k}}$ is the degree that alternative $x_{i}$ should not satisfy the attribute $a_{j}$ at period $t_{k}$, and $0 \leq \mu_{i j, t_{k}}, v_{i j, t_{k}} \leq 1,0 \leq \mu_{i j, t_{k}}+v_{i j, t_{k}} \leq 1$.

\subsection{Analysis of the existing DIF-MADM methods}

Although with some interesting and solid results, there are still some drawbacks found in the existing DIF-MADM methods presented in Gumus [15], Xu [39], Wei [32] and Park [26]. In these DIF-MADM methods, different aggregation operators were introduced. First of all, we recall some operators defined on intuitionistic fuzzy variables [39]

Let $\alpha\left(t_{1}\right)=\left(\mu_{\alpha\left(t_{1}\right)}, v_{\alpha\left(t_{1}\right)}\right), \alpha\left(t_{2}\right)=\left(\mu_{\alpha\left(t_{2}\right)}, v_{\alpha\left(t_{2}\right)}\right)$ be two IFNs, then

(1) $\alpha\left(t_{1}\right) \otimes \alpha\left(t_{2}\right)=\left(\mu_{\alpha\left(t_{1}\right)} \mu_{\alpha\left(t_{2}\right)}, v_{\alpha\left(t_{1}\right)}+v_{\alpha\left(t_{2}\right)}-v_{\alpha\left(t_{1}\right)} v_{\alpha\left(t_{2}\right)}\right)$,

(2) $\alpha\left(t_{1}\right) \oplus \alpha\left(t_{2}\right)=\left(\mu_{\alpha\left(t_{1}\right)}+\mu_{\alpha\left(t_{2}\right)}-\mu_{\alpha\left(t_{1}\right)} \mu_{\alpha\left(t_{2}\right)}, v_{\alpha\left(t_{1}\right)} v_{\alpha\left(t_{2}\right)}\right)$,

(3) $\lambda \alpha\left(t_{1}\right)=\left(1-\left(1-\mu_{\alpha\left(t_{1}\right)}\right)^{\lambda}, v_{\alpha\left(t_{1}\right)}^{\lambda}\right)$,

(4) $\alpha\left(t_{1}\right)^{\lambda}=\left(\mu_{\alpha\left(t_{1}\right)}^{\lambda}, 1-\left(1-v_{\alpha\left(t_{1}\right)}\right)^{\lambda}\right)$.

Base on the above definitions, some aggregation operators are defined as follows:

Let $\alpha\left(t_{1}\right), \alpha\left(t_{2}\right), \cdots, \alpha\left(t_{p}\right)$ be a collection of IFNs collected at $p$ different periods $t_{k}(k=$ $1,2, \cdots, p)$, and $\lambda(t)=\left(\lambda\left(t_{1}\right), \lambda\left(t_{2}\right), \cdots, \lambda\left(t_{p}\right)\right)$ be the weight vector of the periods $t_{k}(k=1,2, \cdots, p)$ with $\lambda\left(t_{i}\right) \geq 0$ and $\sum_{i=1}^{p} \lambda\left(t_{i}\right)=1$. Then a dynamic intuitionistic fuzzy weighted averaging (DIFWA) operator [39] is defined as follows:

$$
\begin{aligned}
\operatorname{DIFWA}\left(\alpha\left(t_{1}\right), \alpha\left(t_{2}\right), \cdots, \alpha\left(t_{p}\right)\right) & =\lambda\left(t_{1}\right) \alpha\left(t_{1}\right) \oplus \lambda\left(t_{2}\right) \alpha\left(t_{2}\right) \oplus \cdots \oplus \lambda\left(t_{p}\right) \alpha\left(t_{p}\right) \\
& \left.=\left(1-\prod_{i=1}^{p}\left(1-\mu_{\alpha\left(t_{i}\right)}\right)^{\lambda\left(t_{i}\right)}, \prod_{i=1}^{p} v_{\alpha\left(t_{i}\right)}\right)^{\lambda\left(t_{i}\right)}\right) ;
\end{aligned}
$$

190 A dynamic intuitionistic fuzzy weighted geometric (DIFWG) operator $[32,26]$ is defined as follows:

$$
\begin{aligned}
\operatorname{DIFWG}\left(\alpha\left(t_{1}\right), \alpha\left(t_{2}\right), \cdots, \alpha\left(t_{p}\right)\right) & =\alpha\left(t_{1}\right)^{\lambda\left(t_{1}\right)} \oplus \alpha\left(t_{2}\right)^{\lambda\left(t_{2}\right)} \oplus \cdots \oplus \alpha\left(t_{p}\right)^{\lambda\left(t_{p}\right)} \\
& \left.=\left(\prod_{i=1}^{p} \mu_{\alpha\left(t_{i}\right)}^{\lambda\left(t_{i}\right)}, 1-\prod_{i=1}^{p}\left(1-v_{\alpha\left(t_{i}\right)}\right)\right)^{\lambda\left(t_{i}\right)}\right) .
\end{aligned}
$$

A dynamic intuitionistic fuzzy Einstein weighted geometric $\left(\mathrm{DIFWG}^{\epsilon}\right)$ operator $[15,28]$ is defined as follows:

$$
\begin{aligned}
& \operatorname{DIFWG}\left(\alpha\left(t_{1}\right), \alpha\left(t_{2}\right), \cdots, \alpha\left(t_{p}\right)\right) \\
& =\left(\frac{2 \prod_{i=1}^{p} \mu_{\alpha\left(t_{i}\right)}^{\lambda\left(t_{i}\right)}}{\prod_{i=1}^{p}\left(2-\mu_{\alpha\left(t_{i}\right)}\right)^{\lambda\left(t_{i}\right)}+\prod_{i=1}^{p} \mu_{\alpha\left(t_{i}\right)}^{\lambda\left(t_{i}\right)}}, \frac{\left.\left.\prod_{i=1}^{p}\left(1+v_{\alpha\left(t_{i}\right)}\right)\right)^{\lambda\left(t_{i}\right)}-\prod_{i=1}^{p}\left(1-v_{\alpha\left(t_{i}\right)}\right)\right)^{\lambda\left(t_{i}\right)}}{\left.\left.\prod_{i=1}^{p}\left(1+v_{\alpha\left(t_{i}\right)}\right)\right)^{\lambda\left(t_{i}\right)}+\prod_{i=1}^{p}\left(1-v_{\alpha\left(t_{i}\right)}\right)\right)^{\lambda\left(t_{i}\right)}}\right) .
\end{aligned}
$$


In the following, we analyse and illustrate some drawbacks about those aggregation opera-

195

(Drawback A.) For Eq. (11), if there exist $\mu_{\alpha\left(t_{1}\right)}=1$, and $\mu_{\alpha\left(t_{2}\right)}=\cdots=\mu_{\alpha\left(t_{p}\right)}=0$, then $1-\prod_{i=1}^{p}\left(1-\mu_{\alpha\left(t_{i}\right)}\right)^{\lambda\left(t_{i}\right)}=1$, which is incorrect because of the impact of the values $\mu_{\alpha\left(t_{2}\right)}=\cdots=$ $\mu_{\alpha\left(t_{p}\right)}=0$ on the aggregation result is not considered. For example, in a DIF-MADM problem, as far as alternative $x_{1}$ is concerned, if $\mu_{\alpha\left(t_{1}\right)}=1$ for the first attribute at the period $t_{1}$, although the

200 degree of memberships and no-memberships of the first attribute regarding to the alternative $x_{1}$ at others periods changed, aggregation result of the first attribute regarding to $x_{1}$ will be constant. Therefore, DIFWA operator Eq. (11) is not well defined. Accordingly the DIF-MADM method presented in [39] uses this ill-defined DIFWA operator will get an unreasonable preference order of the alternatives in some situations.

(Drawback B.) Considering Eq. (12) and Eq. (13), if there is only one membership degree of IFNs is equal to 0 , the aggregation membership degree of IFNs is 0 even if the membership degrees of $n-1$ IFNs are not 0 , this seems a rather extreme property. Therefore, the DIF-MADM methods in [32] and [15] will again lead to inappropriate preference order of alternatives in some situations.

(Drawback C.) besides Drawback B about, there is another drawback in the existing DIFMADM methods presented in [15, 26, 32, 39]. The example below gives a better illustration.

Example 2. Suppose there is an investment company, which wants to invest a sum of money in the best option (adapted from Ref.[32]). There is a panel with three possible alternatives to invest the money: (1) $x_{1}$ is a car company; (2) $x_{2}$ is a food company; (3) $x_{3}$ is a computer company.

${ }_{215}$ The investment company must take a decision according to the following four attributes: (1) $a_{1}$ is the risk analysis; (2) $a_{2}$ is the growth analysis; (3) $a_{3}$ is the social-political impact analysis and the environmental impact analysis. The three possible alternatives $x_{i}(i=1,2,3)$ are to be evaluated using the intuitionistic fuzzy information by the decision maker under the above four attributes at the periods $t_{k}(k=1,2,3)$, as listed in the following matrix, shown as Tabled 1 and

220 2. Let $\lambda(t)=\left(\frac{1}{3}, \frac{1}{3}, \frac{1}{3}\right)$ be the weight vector of the periods $t_{k}(k=1,2,3)$, and $w=\left(\frac{1}{3}, \frac{1}{3}, \frac{1}{3}\right)$ be the weight vector of the attributes $a_{j}(j=1,2,3,4)$.

Table 1: Individual IF decision matrix $D_{t_{k}}(k=1,2,3)$

\begin{tabular}{ccccc}
\hline years & & $a_{1}$ & $a_{2}$ & $a_{3}$ \\
\hline \multirow{4}{*}{$t_{1}$} & $x_{1}$ & $(0.25,0.7)$ & $(0.5,0.2)$ & $(0.7,0.2)$ \\
& $x_{2}$ & $(0.5,0.2)$ & $(0.4,0.5)$ & $(0.4,0.1)$ \\
& $x_{3}$ & $(0.4,0.3)$ & $(0.5,0.3)$ & $(0.6,0.3)$ \\
\hline \multirow{4}{*}{$t_{2}$} & $x_{1}$ & $(0.6,0.3)$ & $(0.4,0.1)$ & $(0.6,0.1)$ \\
& $x_{2}$ & $(0.7,0.1)$ & $(0.25,0.7)$ & $(0.5,0.3)$ \\
& $x_{3}$ & $(0.5,0.2)$ & $(0.7,0.2)$ & $(0.4,0.5)$ \\
\hline \multirow{4}{*}{$t_{3}$} & $x_{1}$ & $(0.4,0.5)$ & $(0.7,0.3)$ & $(0.5,0.3)$ \\
& $x_{2}$ & $(0.6,0.3)$ & $(0.6,0.3)$ & $(0.7,0.2)$ \\
& $x_{3}$ & $(0.7,0.1)$ & $(0.6,0.1)$ & $(0.25,0.7)$ \\
\hline
\end{tabular}

Calculate the distance between the alternative $x_{i}$ and the intuititonistic fuzzy positive ideal solution (IFPIS) $\alpha^{+}=(1,0)$ and the distance between the alternative $x_{i}$ and the intuititonistic fuzzy negative ideal solution (IFNIS) $\alpha^{-}=(0,1)$ by the equations in [39], respectively, we have 
Table 2: Complex intuitionistic fuzzy decision matrix by DIFWA operators

\begin{tabular}{cccc}
\hline$a_{1}$ & $a_{2}$ & $a_{3}$ & \\
\hline$x_{1}$ & $(0.4351,0.4721)$ & $(0.5515,0.1820)$ & $(0.6081,0.1820)$ \\
$x_{2}$ & $(0.6081,0.1820)$ & $(0.4351,0.4721)$ & $(0.5515,0.1820)$ \\
$x_{3}$ & $(0.5515,0.1820)$ & $(0.6081,0.1820)$ & $(0.4351,0.4721)$ \\
\hline
\end{tabular}

$$
\begin{aligned}
& d\left(x_{1}, \alpha^{+}\right)=d\left(x_{2}, \alpha^{+}\right)=d\left(x_{3}, \alpha^{+}\right)=0.4684, \\
& d\left(x_{1}, \alpha^{-}\right)=d\left(x_{2}, \alpha^{-}\right)=d\left(x_{3}, \alpha^{-}\right)=0.7213 .
\end{aligned}
$$

According to [39], the closeness coefficient of each alternative is given by

$$
c\left(x_{i}\right)=\frac{d\left(x_{i}, \alpha^{-}\right)}{d\left(x_{i}, \alpha^{-}\right)+d\left(x_{i}, \alpha^{+}\right)} .
$$

It follows that $c\left(x_{1}\right)=c\left(x_{2}\right)=c\left(x_{3}\right)=0.6063$. Therefore $x_{1}=x_{2}=x_{3}$, which is obviously an incorrect preference orders of alternatives. The same results also can be obtained by using Gumus's [15] and Wei's [32] DIF-MADM method based on the DIFWG operators defined in Eqs. (12) and (13).

In Section 4 bellow, two new methods are proposed to overcome the above mentioned drawbacks of the existing DIF-MADM methods.

\section{New methods for DIF-MADM problems}

In this section, we propose two kinds of DIF-MADM methods to overcome the drawbacks presented in Section 3. It shows that Method I can overcome the drawbacks A, B and C. And Method II can overcome the drawbacks B and C.

\subsection{Method I: New DIF-MADM based on the ER methodology}

Suppose that the alternatives are assessed on each attribute using the following two assessment grades: $H_{1}$ and $H_{2}$, where $H_{1}$ stands for satisfying the fuzzy concept "excellence", $H_{2}$ stands for not satisfying the fuzzy concept "excellence", and $H=\left\{H_{1}, H_{2}\right\}$ stands for the assessment grade indeterminacy. The proposed method for intuitionistic fuzzy MADM based on IFSs and the ER algorithm is now presented as follows:

Step 1. Determine the belief matrix of decision maker w.r.t. attribute $a_{j}$ of alternative $x_{i}$ regarding the evaluation grade $H_{1}, H_{2}$ as follows:

$$
\begin{aligned}
& D_{t_{k}}=\left(\mu_{i j, t_{k}}, v_{i j, t_{k}}\right)_{m \times n}=\left(\beta_{1 j, t_{k}}\left(x_{i}\right), \beta_{2 j, t_{k}}\left(x_{i}\right)\right)_{m \times n} \\
& =\left(\begin{array}{cccc}
\left(\beta_{11, t_{k}}\left(x_{1}\right), \beta_{21, t_{k}}\left(x_{1}\right)\right) & \left(\beta_{12, t_{k}}\left(x_{1}\right), \beta_{22, t_{k}}\left(x_{1}\right)\right) & \cdots & \left(\beta_{1 n, t_{k}}\left(x_{1}\right), \beta_{2 n, t_{k}}\left(x_{1}\right)\right) \\
\left(\beta_{11, t_{k}}\left(x_{2}\right), \beta_{21, t_{k}}\left(x_{2}\right)\right) & \left(\beta_{12, t_{k}}\left(x_{2}\right), \beta_{22, t_{k}}\left(x_{2}\right)\right) & \cdots & \left(\beta_{1 n, t_{k}}\left(x_{2}\right), \beta_{2 n, t_{k}}\left(x_{2}\right)\right) \\
\vdots & \vdots & \vdots & \vdots \\
\left(\beta_{11, t_{k}}\left(x_{m}\right), \beta_{21, t_{k}}\left(x_{m}\right)\right) & \left(\beta_{12, t_{k}}\left(x_{m}\right), \beta_{22, t_{k}}\left(x_{m}\right)\right) & \cdots & \left(\beta_{1 n, t_{k}}\left(x_{m}\right), \beta_{2 n, t_{k}}\left(x_{m}\right)\right)
\end{array}\right)
\end{aligned}
$$

where $\left(\mu_{i j, t_{k}}, v_{i j, t_{k}}\right)=\left(\beta_{1 j, t_{k}}\left(x_{i}\right), \beta_{2 j, t_{k}}\left(x_{i}\right)\right), \beta_{1 j, t_{k}}\left(x_{i}\right)$ denotes the degree of belief of decision maker $d_{l}$ w.r.t. attribute $a_{j}$ of alternative $x_{i}$ at period $t_{k}$ regarding evaluation grade $H_{1}$ and $\beta_{2 j, t_{k}}\left(x_{i}\right)$ 
represents the degree of belief w. r. t. attribute $a_{j}$ of alternative $x_{i}$ at period $t_{k}$ regarding evaluation grade $H_{2}, 0 \leq \beta_{1 j, t_{k}}\left(x_{i}\right), \beta_{2 j, t_{k}}\left(x_{i}\right) \leq 1$ and $0 \leq \beta_{1 j, t_{k}}\left(x_{i}\right)+\beta_{2 j, t_{k}}\left(x_{i}\right) \leq 1(j=1,2, \cdots, n ; i=$ $, 2, \cdots, m ; k=1,2, \cdots, p)$.

Step 1.1. Based on the above step, the intuitionistic fuzzy assessment $\left(\mu_{i j, t_{k}}, v_{i j, t_{k}}\right)$ can be transformed into the ER belief distribution assessment profiled as

$$
\left\{\left(H_{1}, \beta_{1 j, t_{k}}\left(x_{i}\right)\right),\left(H_{2}, \beta_{2 j, t_{k}}\left(x_{i}\right)\right),\left(H, \beta_{H j, t_{k}}\left(x_{i}\right)\right)\right\}
$$

Transform the degree of belief $\beta_{q j, t_{k}}\left(x_{i}\right)$ into basic probability mass $\tilde{m}_{q j, t_{k}}\left(x_{i}\right)$ and $\tilde{m}_{H_{j}}\left(x_{i}\right)$ by 250 the following formulae [49]:

$$
\begin{aligned}
& \tilde{m}_{q j, t_{k}}\left(x_{i}\right)=w_{j}\left(t_{k}\right) \beta_{q j, t_{k}}\left(x_{i}\right) \\
& \tilde{m}_{H j, t_{k}}\left(x_{i}\right)=1-\sum_{j=1}^{n} \tilde{m}_{q j, t_{k}}\left(x_{i}\right) .
\end{aligned}
$$

We can then obtain the basic probability mass matrix:

$$
P_{t_{k}}=\left(\begin{array}{cccc}
\left(\tilde{m}_{11, t_{k}}\left(x_{1}\right), \tilde{m}_{21, t_{k}}\left(x_{1}\right)\right) & \left(\tilde{m}_{12, t_{k}}\left(x_{1}\right), \tilde{m}_{22, t_{k}}\left(x_{1}\right)\right) & \cdots & \left(\tilde{m}_{1 n, t_{k}}\left(x_{1}\right), \tilde{m}_{2 n, t_{k}}\left(x_{1}\right)\right) \\
\left(\tilde{m}_{11, t_{k}}\left(x_{2}\right), \tilde{m}_{21, t_{k}}\left(x_{2}\right)\right) & \left(\tilde{m}_{12, t_{k}}\left(x_{2}\right), \tilde{m}_{22, t_{k}}\left(x_{2}\right)\right) & \cdots & \left(\tilde{m}_{1 n, t_{k}}\left(x_{2}\right), \tilde{m}_{2 n, t_{k}}\left(x_{2}\right)\right) \\
\vdots & \vdots & \vdots & \vdots \\
\left(\tilde{m}_{11, t_{k}}\left(x_{m}\right), \tilde{m}_{21, t_{k}}\left(x_{m}\right)\right) & \left(\tilde{m}_{12, t_{k}}\left(x_{m}\right), \tilde{m}_{22, t_{k}}\left(x_{m}\right)\right) & \cdots & \left(\tilde{m}_{1 n, t_{k}}\left(x_{m}\right), \tilde{m}_{2 n, t_{k}}\left(x_{m}\right)\right)
\end{array}\right)
$$

where $0 \leq \tilde{m}_{1 j, t_{k}}\left(x_{i}\right), \tilde{m}_{2 j, t_{k}}\left(x_{i}\right) \leq 1$ and $0 \leq \tilde{m}_{1 j, t_{k}}\left(x_{i}\right)+\tilde{m}_{2 j, t_{k}}\left(x_{i}\right) \leq 1(j=1,2, \cdots, n ; i=$ $1,2, \cdots, m ; k=1,2, \cdots, p)$.

Step 1.2. Let the combined probability mass $\tilde{n}_{q 1, t_{k}}\left(x_{i}\right)$ of the decision maker $d_{l}$ w.r.t. attribute $a_{j}$ of alternative $x_{i}$ at period $t_{k}$ be equal to $\tilde{m}_{q 1, t_{k}}\left(x_{i}\right)$, that is, $\tilde{n}_{q 1, t_{k}}\left(x_{i}\right)=\tilde{m}_{q 1, t_{k}}\left(x_{i}\right)(q=1,2)$. Similarly, $\tilde{n}_{H 1, t_{k}}\left(x_{i}\right)=\tilde{m}_{H 1, t_{k}}\left(x_{i}\right)(q=1,2)$. Now, calculate the combined probability mass $\tilde{n}_{q j, t_{k}}\left(x_{i}\right)$ and $\tilde{n}_{H j, t_{k}}\left(x_{i}\right)$ w. r. t. the attribute $a_{j}$ of alternative $x_{i}$ at period $t_{k}$ by the following equations:

$$
\begin{aligned}
& \tilde{n}_{q j, t_{k}}\left(x_{i}\right)=\frac{\tilde{n}_{q j-1, t_{k}}\left(x_{i}\right) \tilde{m}_{q j, t_{k}}\left(x_{i}\right)+\tilde{n}_{q j-1, t_{k}}\left(x_{i}\right) \tilde{m}_{H j, t_{k}}\left(x_{i}\right)+\tilde{n}_{H j-1, t_{k}}\left(x_{i}\right) \tilde{m}_{q j, t_{k}}\left(x_{i}\right)}{1-\sum_{r=1}^{2} \sum_{h=1, h \neq r}^{2} \tilde{n}_{r j-1, t_{k}}\left(x_{i}\right) \tilde{m}_{h j, t_{k}}\left(x_{i}\right)} \\
& \tilde{n}_{H j, t_{k}}^{g}\left(x_{i}\right)=\frac{\tilde{n}_{H j-1, t_{k}}\left(x_{i}\right) \tilde{m}_{H j, t_{k}}\left(x_{i}\right)}{1-\sum_{r=1}^{2} \sum_{h=1, h \neq r}^{2} \tilde{n}_{r j-1, t_{k}}\left(x_{i}\right) \tilde{m}_{h j, t_{k}}\left(x_{i}\right)}
\end{aligned}
$$

where $j=1,2, \cdots, n ; i=1,2, \cdots, m ; k=1,2, \cdots, p$.

Step 1.3. Aggregate the evaluating values of decision makers with respect to attribute $a_{j}$ of alternative $x_{i}$ at period $t_{k}$ to obtain the belief:

$$
\beta_{q, t_{k}}\left(x_{i}\right)=\frac{\left(1-\beta_{H, t_{k}}\left(x_{i}\right)\right) \tilde{n}_{q n, t_{k}}\left(x_{j}\right)}{1-\tilde{n}_{H n, t_{k}}\left(x_{i}\right)}
$$

260 with $\beta_{H n, t_{k}}\left(x_{i}\right)=\sum_{j=1}^{n} w_{j}\left(t_{k}\right)\left(1-\sum_{q=1}^{2} \tilde{n}_{q j, t_{k}}\left(x_{i}\right)\right)$. where $\sum_{q=1}^{2} \beta_{q, t_{k}}\left(x_{i}\right)+\beta_{H n, t_{k}}\left(x_{i}\right)=1$. Let the aggregated value obtained by above equations $\beta_{1, t_{k}}\left(x_{i}\right), \beta_{2, t_{k}}\left(x_{i}\right)$ form an intuitionistic fuzzy value $\left(\beta_{1, t_{k}}\left(x_{i}\right), \beta_{2, t_{k}}\left(x_{i}\right)\right)$, where $\beta_{1, t_{k}}\left(x_{i}\right)$ and $\beta_{2, t_{k}}\left(x_{i}\right)$ are the degree of decision maker $d_{l}$ w.r.t. alternative $x_{i}$ at period $t_{k}$ regarding evaluation grades $H_{1}$ and $H_{2}$. 
Step 2. Based on Step 1, construct the aggregated decision making matrix $Q$ as follows

$$
\begin{aligned}
Q & =\left(\beta_{1, t_{k}}\left(x_{i}\right), \beta_{2, t_{k}}\left(x_{i}\right)\right)_{m \times p} \\
& =\left(\begin{array}{cccc}
\left(\beta_{1, t_{1}}\left(x_{1}\right), \beta_{2, t_{1}}\left(x_{1}\right)\right) & \left(\beta_{1, t_{2}}\left(x_{1}\right), \beta_{2, t_{2}}\left(x_{1}\right)\right) & \cdots & \left(\beta_{1, t_{p}}\left(x_{1}\right), \beta_{2, t_{p}}\left(x_{1}\right)\right) \\
\left(\beta_{1, t_{1}}\left(x_{2}\right), \beta_{2, t_{1}}\left(x_{2}\right)\right) & \left(\beta_{1, t_{2}}\left(x_{2}\right), \beta_{2, t_{2}}\left(x_{2}\right)\right) & \cdots & \left(\beta_{1, t_{p}}\left(x_{2}\right), \beta_{2, t_{p}}\left(x_{2}\right)\right) \\
\vdots & \vdots & \vdots & \vdots \\
\left(\beta_{1, t_{1}}\left(x_{m}\right), \beta_{2, t_{1}}\left(x_{m}\right)\right) & \left(\beta_{1, t_{2}}\left(x_{m}\right), \beta_{2, t_{2}}\left(x_{m}\right)\right) & \cdots & \left(\beta_{1, t_{p}}\left(x_{m}\right), \beta_{2, t_{p}}\left(x_{m}\right)\right)
\end{array}\right)
\end{aligned}
$$

where $\left(\beta_{1, t_{k}}\left(x_{i}\right), \beta_{2, t_{k}}\left(x_{i}\right)\right)$ is an IFN, $\beta_{1, t_{k}}\left(x_{i}\right)$ is the degree of belief with respect to alternative

$x_{i}$ at period $t_{k}$ regarding evaluation grade $H_{1}$ and $\beta_{2, t_{k}}\left(x_{i}\right)$ represents the degree of belief $\mathrm{w} . \mathrm{r}$ t. alternative $x_{i}$ at period $t_{k}$ regarding evaluation grade $H_{2}, 0 \leq \beta_{1, t_{k}}\left(x_{i}\right), \beta_{2, t_{k}}\left(x_{i}\right) \leq 1$ and $0 \leq$ $\beta_{1, t_{k}}\left(x_{i}\right)+\beta_{2, t_{k}}\left(x_{i}\right) \leq 1(i=1,2, \cdots, m ; k=1,2, \cdots, p)$.

Step 2.1. Based on the above step, the intuitionistic fuzzy assessment $\left(\beta_{1, t_{k}}\left(x_{i}\right), \beta_{2, t_{k}}\left(x_{i}\right)\right)$ can be transformed into the ER belief distribution assessment profiled by

$$
\left\{\left(H_{1}, \beta_{1}\left(x_{i}\right)\right),\left(H_{2}, \beta_{2}\left(x_{i}\right)\right),\left(H, \beta_{H}\left(x_{i}\right)\right)\right\} .
$$

Transform the degree of belief $\beta_{q, t_{k}}\left(x_{i}\right)$ into basic probability mass $\tilde{m}_{q, t_{k}}\left(x_{i}\right)$ and $\tilde{m}_{H, t_{k}}\left(x_{i}\right)$ by the following formulae:

$$
\begin{aligned}
& \tilde{m}_{q, t_{k}}\left(x_{i}\right)=w_{k} \beta_{q, t_{k}}\left(x_{i}\right) ; \\
& \tilde{m}_{H, t_{k}}\left(x_{i}\right)=1-\sum_{k=1}^{p} \tilde{m}_{q, t_{k}}\left(x_{i}\right) .
\end{aligned}
$$

We can obtain the basic probability mass matrix

$$
Q=\left(\begin{array}{cccc}
\left(\tilde{m}_{1, t_{1}}\left(x_{1}\right), \tilde{m}_{2, t_{1}}\left(x_{1}\right)\right) & \left(\tilde{m}_{1, t_{2}}\left(x_{1}\right), \tilde{m}_{2, t_{2}}\left(x_{1}\right)\right) & \cdots & \left(\tilde{m}_{1, t_{p}}\left(x_{1}\right), \tilde{m}_{2, t_{p}}\left(x_{1}\right)\right) \\
\left(\tilde{m}_{1, t_{1}}\left(x_{2}\right), \tilde{m}_{2, t_{1}}\left(x_{2}\right)\right) & \left(\tilde{m}_{1, t_{2}}\left(x_{2}\right), \tilde{m}_{2, t_{2}}\left(x_{2}\right)\right) & \cdots & \left(\tilde{m}_{1, t_{p}}\left(x_{2}\right), \tilde{m}_{2, t_{p}}\left(x_{2}\right)\right) \\
\vdots & \vdots & \vdots & \vdots \\
\left(\tilde{m}_{1, t_{1}}\left(x_{m}\right), \tilde{m}_{2, t_{1}}\left(x_{m}\right)\right) & \left(\tilde{m}_{1, t_{2}}\left(x_{m}\right), \tilde{m}_{2, t_{2}}\left(x_{m}\right)\right) & \cdots & \left(\tilde{m}_{1, t_{p}}\left(x_{m}\right), \tilde{m}_{2, t_{p}}\left(x_{m}\right)\right)
\end{array}\right)
$$

where $0 \leq \tilde{m}_{1, t_{k}}\left(x_{i}\right), \tilde{m}_{2, t_{k}}\left(x_{i}\right) \leq 1$ and $0 \leq \tilde{m}_{1, t_{k}}\left(x_{i}\right)+\tilde{m}_{2, t_{k}}\left(x_{i}\right) \leq 1(i=1,2, \cdots, m ; k=1,2, \cdots, p)$.

Step 2.2. Let the combined probability mass $\tilde{n}_{q, t_{1}}\left(x_{i}\right)$ w.r.t. alternative $x_{i}$ at period $t_{1}$ be equal to $\tilde{m}_{q, t_{1}}\left(x_{i}\right)$, that is, $n_{q, t_{1}}\left(x_{i}\right)=\tilde{m}_{q, t_{1}}\left(x_{i}\right)(q=1,2)$. Similarly, $n_{C, t_{1}}\left(x_{i}\right)=\tilde{m}_{C, t_{1}}\left(x_{i}\right)(q=1,2)$. Now, calculate the combined probability mass $\tilde{n}_{q, t_{k}}\left(x_{i}\right)$ and $\tilde{n}_{C, t_{k}}\left(x_{i}\right)$ w. r. t. alternative $x_{i}$ at period $t_{k}$ by the following equations:

$$
\begin{aligned}
& \tilde{n}_{q, t_{k}}\left(x_{i}\right)=\frac{\tilde{n}_{q, t_{k-1}}\left(x_{i}\right) \tilde{m}_{q, t_{k}}\left(x_{i}\right)+\tilde{n}_{q, t_{k-1}}\left(x_{i}\right) \tilde{m}_{H, t_{k}}\left(x_{i}\right)+\tilde{n}_{H, t_{k-1}}\left(x_{i}\right) \tilde{m}_{q, t_{k}}\left(x_{i}\right)}{1-\sum_{r=1}^{2} \sum_{h=1, h \neq r}^{2} \tilde{n}_{r, t_{k-1}}\left(x_{i}\right) \tilde{m}_{h, t_{k}}\left(x_{i}\right)} \\
& \tilde{n}_{H, t_{k}}\left(x_{i}\right)=\frac{\tilde{n}_{H, t_{k-1}}\left(x_{i}\right) \tilde{m}_{H, t_{k}}\left(x_{i}\right)}{1-\sum_{r=1}^{2} \sum_{h=1, h \neq r}^{2} \tilde{n}_{r, t_{k-1}}\left(x_{i}\right) \tilde{m}_{h, t_{k}}\left(x_{i}\right)},
\end{aligned}
$$

where $k=2, \cdots, p ; i=1,2, \cdots, m$.

Step 2.3. Aggregate the evaluating values of decision makers with respect to alternative $x_{i}$ to obtain the belief:

$$
\beta_{q}\left(x_{i}\right)=\frac{\left(1-\beta_{H}\left(x_{i}\right)\right) \tilde{n}_{q}\left(x_{j}\right)}{1-\tilde{n}_{H}\left(x_{i}\right)}, q=1,2 .
$$


280 with $\beta_{H}\left(x_{i}\right)=\sum_{k=1}^{p} w_{k}\left(1-\sum_{q=1}^{2} \tilde{n}_{q, t_{k}}\left(x_{i}\right)\right)$. where $\sum_{q=1}^{2} \beta_{q}\left(x_{i}\right)+\beta_{H}\left(x_{i}\right)=1$. Let the aggregated value be obtained by above equations $\beta_{1}\left(x_{i}\right), \beta_{2}\left(x_{i}\right)$ form an IFN $\left(\beta_{1}\left(x_{i}\right), \beta_{2}\left(x_{i}\right)\right)$, where $\beta_{1}\left(x_{i}\right)$ and $\beta_{2}\left(x_{i}\right)$ are the degree decision makers of alternative $x_{j}$ regarding evaluation grades $H_{1}$ and $H_{2}$, respectively.

Step 3 Calculate the scores of IFN $\left(\beta_{1}\left(x_{i}\right), \beta_{2}\left(x_{i}\right)\right)$ obtained by the aggregation result of Step 285 3. Let $\alpha_{i}=\left(\beta_{1}\left(x_{i}\right), \beta_{2}\left(x_{i}\right)\right)(i=1,2, \cdots, m)$.

Step 3.1 According to Eq. (1), calculate $H\left(\alpha_{i}\right)$ and $H\left(\alpha_{i}^{C}\right)$, where $\alpha_{i}^{C}=\left(\beta_{2}\left(x_{i}\right), \beta_{1}\left(x_{i}\right)\right),(i=$ $1,2, \cdots, m)$;

Step 3.2 Calculate the score of $\alpha_{i}$ and denote as $S\left(\alpha_{i}\right)$;

Step 4 Determine the ranking of alternatives according to Step 3.2. The larger the value $S\left(\alpha_{i}\right)$, the better the order of alternative $x_{i}(i=1,2, \cdots, m)$.

Step 5 End.

\subsection{Method II: New DIF-MADM method based on new aggregation operators}

In this section, we propose new operators in IFNs and further propose the new DIFWG operator, and then introduce a new DIF-MADM method which can overcome some drawbacks analysed in Section 3.

\subsubsection{New aggregation operators for DIF-MADM problems}

Before the new operator of IFNs are given, we firstly introduce a new definition of operation on intuitionistic fuzzy variables.

Definition 5. Let $\alpha\left(t_{1}\right)=\left(\mu_{\alpha\left(t_{1}\right)}, v_{\alpha\left(t_{1}\right)}\right), \alpha\left(t_{2}\right)=\left(\mu_{\alpha\left(t_{2}\right)}, v_{\alpha\left(t_{2}\right)}\right)$ be two IFNs. Then

(1) $\alpha\left(t_{1}\right) \otimes \alpha\left(t_{2}\right)=\left(\mu_{\alpha\left(t_{1}\right)}+\mu_{\alpha\left(t_{2}\right)}-\mu_{\alpha\left(t_{1}\right)} \mu_{\alpha\left(t_{2}\right)}, v_{\alpha\left(t_{1}\right)}\left(1-\mu_{\alpha\left(t_{2}\right)}-v_{\alpha\left(t_{2}\right)}\right)+v_{\alpha\left(t_{2}\right)}\left(1-\mu_{\alpha\left(t_{1}\right)}\right)\right)$,

(2) $\alpha\left(t_{1}\right)^{\lambda}=\left(1-\left(1-\mu_{\alpha\left(t_{1}\right)}\right)^{\lambda},\left(1-\mu_{\alpha\left(t_{1}\right)}\right)^{\lambda}-\left(1-\mu_{\alpha\left(t_{1}\right)}-v_{\alpha\left(t_{1}\right)}\right)^{\lambda}\right)$.

Base on the above operators, a modified DIFWG aggregation operator is defined as follows:

Definition 6. Let $\alpha\left(t_{1}\right), \alpha\left(t_{2}\right), \cdots, \alpha\left(t_{p}\right)$ be a collection of IFNs collected at p different periods $t_{k}(k=1,2, \cdots, p)$, and $\lambda(t)=\left(\lambda\left(t_{1}\right), \lambda\left(t_{2}\right), \cdots, \lambda\left(t_{p}\right)\right)$ be the weight vector of the periods $t_{k}(k=$ $1,2, \cdots, p)$ with $\lambda\left(t_{i}\right) \geq 0$ and $\sum_{i=1}^{p} \lambda\left(t_{i}\right)=1$. Then a modified dynamic intuitionistic fuzzy weighted geometric (MDIFWG) operator is defined as follows:

$$
\operatorname{MDIFWG}\left(\alpha\left(t_{1}\right), \alpha\left(t_{2}\right), \cdots, \alpha\left(t_{p}\right)\right)=\alpha\left(t_{1}\right)^{\lambda\left(t_{1}\right)} \oplus \alpha\left(t_{2}\right)^{\lambda\left(t_{2}\right)} \oplus \cdots \oplus \alpha\left(t_{p}\right)^{\lambda\left(t_{p}\right)} .
$$

Based on (1), (2) in Definition 3, we have:

$$
\begin{aligned}
& \operatorname{MDIFWG}\left(\alpha\left(t_{1}\right), \alpha\left(t_{2}\right), \cdots, \alpha\left(t_{p}\right)\right) \\
& \left.=\left(1-\prod_{i=1}^{p}\left(1-\mu_{\alpha\left(t_{i}\right)}\right)\right)^{\lambda\left(t_{i}\right)}, \prod_{i=1}^{p}\left(1-\mu_{\alpha\left(t_{i}\right)}\right)^{\lambda\left(t_{i}\right)}-\prod_{i=1}^{p}\left(1-\mu_{\alpha\left(t_{i}\right)}-v_{\alpha\left(t_{i}\right)}\right)^{\lambda\left(t_{i}\right)}\right) .
\end{aligned}
$$

\subsection{New DIF-MADM method based on MDIFWG operators}

In this section, we design a new method for DIF-MADM based on the proposed MDIFWG operator presented in Section 4.1.1. The details of this method are described as follows: 
Step 1. Utilize the MDIFWG operator to aggregate all the intuitionistic fuzzy decision matrices $D_{t_{k}}=\left(\alpha_{i j, t_{k}}\right)_{m \times n}(k=1,2, \cdots, p)$ into a complex intuitionistic fuzzy decision matrix $D=\left(\alpha_{i j}\right)_{m \times n}$ :

$$
\begin{aligned}
\alpha_{i j} & =\operatorname{MDIFWG}\left(\left(\mu_{i j, t_{1}}, v_{i j, t_{1}}\right),\left(\mu_{i j, t_{2}}, v_{i j, t_{2}}\right), \cdots,\left(\mu_{i j, t_{p}}, v_{i j, t_{p}}\right)\right) \\
& \left.=\left(1-\prod_{k=1}^{p}\left(1-\mu_{i j, t_{k}}\right)\right)^{\lambda\left(t_{k}\right)}, \prod_{k=1}^{p}\left(1-\mu_{i j, t_{k}}\right)^{\lambda\left(t_{k}\right)}-\prod_{k=1}^{p}\left(1-\mu_{i j, t_{k}}-v_{i j, t_{k}}\right)^{\lambda\left(t_{k}\right)}\right),
\end{aligned}
$$

where $\alpha_{i j}=\left(\mu_{i j}, v_{i j}\right)$ is an IFN obtained by Eq. (30) or Eq. (31).

Step 2- Step 6 are the same as Xu's method [39].

Step 2. Define the intuitionistic fuzzy ideal solution (IFIS) $\alpha^{+}=\left(\alpha_{1}^{+}, \cdots, \alpha_{m}^{+}\right)$and the intuitionistic fuzzy negative ideal solution (IFNIS) $\alpha^{-}=\left(\alpha_{1}^{-}, \cdots, \alpha_{m}^{-}\right)$, respectively, where $\alpha_{i}^{+}=$ $(1,0)(i=1,2, \cdots, n)$ are the $n$ largest IFNs and $\alpha_{i}^{+}=(0,1)(i=1,2, \cdots, n)$ are the $n$ smallest IFNs. Furthermore, for convenience, we denote the alternatives $x_{i}(i=1,2, \cdots, m)$ by $x_{i}=\left(\alpha_{i 1}, \alpha_{i 2}, \cdots, \alpha_{i n}\right), i=1,2, \ldots, m$.

Step 3. Calculate the distance between the alternative $x_{i}$ and the IFIS $\alpha^{+}$and the distance between the alternative $x_{i}$ and the IFNIS $\alpha^{-}$, respectively:

$$
\begin{aligned}
& d\left(x_{i}, \alpha^{+}\right)=\sum_{j=1}^{n} w_{j}\left(1-\mu_{i j}\right), \\
& d\left(x_{i}, \alpha^{-}\right)=\sum_{j=1}^{n} w_{j}\left(1-v_{i j}\right) .
\end{aligned}
$$

Step 4. Calculate the closeness coefficient of each alternative

$$
c\left(x_{i}\right)=\frac{d\left(x_{i}, \alpha^{-}\right)}{d\left(x_{i}, \alpha^{+}\right)+d\left(x_{i}, \alpha^{-}\right)} .
$$

Step 5. Determine the preference orders of all the alternatives $x_{i}(i=1,2, \cdots, m)$ according to the closeness coefficients $c\left(x_{i}\right)(i=1,2, \cdots, n)$, the greater the value $c\left(x_{i}\right)$, the better the alternative $x_{i}$.

Step 6. End.

\section{Case study}

In this section, we use some examples to illustrate and compare the proposed methods with some existing DIF-MADM methods.

\subsection{Examples and comparative analysis}

Example 3. A problem of evaluating university faculty for tenure and promotion (adapted from

335 Bryson and Mobolurin [26]) is used to illustrate the developed approach. A practical use of the proposed approach involves the evaluation of university faculty for tenure and promotion. The attributes at some university are $A_{1}$ : teaching, $A_{2}$ : research, and $A_{3}$ : service. The committee evaluates the performance of five faculty candidates (alternatives) $x_{i}(i=1,2,3,4,5)$ in the three years $t_{k}(j=1,2,3)$. According to the attribute $G_{j}(j=1,2,3)$, and construct, respectively, the intuitionistic fuzzy decision matrices $D_{t_{k}}(k=1,2,3)$. Let $\lambda(t)=(0.2,0.3,0.5)$ be the weight vector of the years $t_{k}$ and $w=(0.3,0.4,0.3)$ be the weight vector of the attributes $A_{j}(j=1,2,3)$

(1) Method I: We utilize the ER algorithm. 
Table 3: Individual IF decision matrix $D_{t_{k}}(k=1,2,3)$

\begin{tabular}{ccccc}
\hline years & & $a_{1}$ & $a_{2}$ & $a_{3}$ \\
\hline \multirow{4}{*}{$t_{1}$} & $x_{1}$ & $(0.8,0.1)$ & $(0.9,0.1)$ & $(0.7,0.2)$ \\
& $x_{2}$ & $(0.7,0.3)$ & $(0.6,0.2)$ & $(0.6,0.3)$ \\
& $x_{3}$ & $(0.5,0.4)$ & $(0.7,0.3)$ & $(0.6,0.3)$ \\
& $x_{4}$ & $(0.9,0.1)$ & $(0.7,0.2)$ & $(0.8,0.2)$ \\
& $x_{5}$ & $(0.6,0.1)$ & $(0.8,0.2)$ & $(0.50 .1)$ \\
\hline \multirow{4}{*}{$t_{2}$} & $x_{1}$ & $(0.9,0.1)$ & $(0.8,0.2)$ & $(0.8,0.1)$ \\
& $x_{2}$ & $(0.8,0.2)$ & $(0.5,0.1)$ & $(0.7,0.2)$ \\
& $x_{3}$ & $(0.5,0.5)$ & $(0.7,0.2)$ & $(0.8,0.2)$ \\
& $x_{4}$ & $(0.9,0.1)$ & $(0.9,0.1)$ & $(0.7,0.3)$ \\
& $x_{5}$ & $(0.5,0.2)$ & $(0.6,0.3)$ & $(0.6,0.2)$ \\
\hline \multirow{4}{*}{$t_{3}$} & $x_{1}$ & $(0.7,0.1)$ & $(0.9,0.1)$ & $(0.9,0.1)$ \\
& $x_{2}$ & $(0.9,0.1)$ & $(0.6,0.2)$ & $(0.5,0.2)$ \\
& $x_{3}$ & $(0.4,0.5)$ & $(0.8,0.1)$ & $(0.7,0.1)$ \\
& $x_{4}$ & $(0.8,0.1)$ & $(0.7,0.2)$ & $(0.9,0.1)$ \\
& $x_{5}$ & $(0.6,0.3)$ & $(0.8,0.2)$ & $(0.7,0.2)$ \\
\hline
\end{tabular}

Step 1.1: Based on the decision matrices $D_{t_{1}}, D_{t_{2}}$ and $D_{t_{3}}$. Based on the weight of attributes and Eq. (15), Eq. (16), we can obtain the basic probability mass $P_{t_{1}}, P_{t_{2}}$ and $P_{t_{3}}$ :

$$
\begin{gathered}
P_{t_{1}}=\left(\begin{array}{lll}
(0.24,0.03) & (0.36,0.04) & (0.21,0.06) \\
(0.21,0.09) & (0.24,0.08) & (0.18,0.09) \\
(0.15,0.12) & (0.28,0.12) & (0.18,0.09) \\
(0.27,0.03) & (0.28,0.08) & (0.24,0.06) \\
(0.18,0.03) & (0.32,0.08) & (0.15,0.03)
\end{array}\right) \\
P_{t_{2}}=\left(\begin{array}{lll}
(0.27,0.03) & (0.32,0.08) & (0.24,0.03) \\
(0.24,0.06) & (0.2,0.04) & (0.21,0.06) \\
(0.15,0.15) & (0.28,0.08) & (0.24,0.06) \\
(0.27,0.03) & (0.36,0.04) & (0.21,0.09) \\
(0.15,0.06) & (0.24,0.12) & (0.18,0.06)
\end{array}\right) \\
P_{t_{3}}=\left(\begin{array}{lll}
(0.21,0.03) & (0.36,0.04) & (0.27,0.03) \\
(0.27,0.03) & (0.24,0.08) & (0.15,0.06) \\
(0.12,0.15) & (0.32,0.04) & (0.21,0.03) \\
(0.24,0.03) & (0.28,0.08) & (0.27,0.03) \\
(0.18,0.09) & (0.32,0.08) & (0.21,0.06)
\end{array}\right)
\end{gathered}
$$


Step 1.2 We can obtain the combined probability based on Eq. (17)

$$
\begin{aligned}
& \tilde{n}_{13, t_{1}}\left(x_{1}\right)=0.5911, \tilde{n}_{23, t_{1}}\left(x_{1}\right)=0.0686, \tilde{n}_{13, t_{1}}\left(x_{2}\right)=0.457, \tilde{n}_{23, t_{1}}\left(x_{2}\right)=0.1598, \\
& \tilde{n}_{13, t_{1}}\left(x_{3}\right)=0.4341, \tilde{n}_{23, t_{1}}\left(x_{3}\right)=0.2053, \tilde{n}_{13, t_{1}}\left(x_{4}\right)=0.568, \tilde{n}_{23, t_{1}}\left(x_{4}\right)=0.0928, \\
& \tilde{n}_{13, t_{1}}\left(x_{5}\right)=0.5016, \tilde{n}_{23, t_{1}}\left(x_{5}\right)=0.0896, \tilde{n}_{13, t_{2}}\left(x_{1}\right)=0.5971, \tilde{n}_{23, t_{2}}\left(x_{1}\right)=0.0755, \\
& \tilde{n}_{13, t_{2}}\left(x_{2}\right)=0.4891, \tilde{n}_{23, t_{2}}\left(x_{2}\right)=0.0978, \tilde{n}_{13, t_{2}}\left(x_{3}\right)=0.4754, \tilde{n}_{23, t_{2}}\left(x_{3}\right)=0.1709, \\
& \tilde{n}_{13, t_{2}}\left(x_{4}\right)=0.5998, \tilde{n}_{23, t_{4}}\left(x_{4}\right)=0.0814, \tilde{n}_{13, t_{2}}\left(x_{5}\right)=0.4261, \tilde{n}_{23, t_{2}}\left(x_{5}\right)=0.1576, \\
& \tilde{n}_{13, t_{3}}\left(x_{1}\right)=0.6128, \tilde{n}_{23, t_{3}}\left(x_{1}\right)=0.0523, \tilde{n}_{13, t_{3}}\left(x_{2}\right)=0.4953, \tilde{n}_{23, t_{3}}\left(x_{2}\right)=0.1022, \\
& \tilde{n}_{13, t_{3}}\left(x_{3}\right)=0.48, \tilde{n}_{23, t_{3}}\left(x_{3}\right)=0.1294, \tilde{n}_{13, t_{3}}\left(x_{4}\right)=0.5742, \tilde{n}_{23, t_{4}}\left(x_{2}\right)=0.0772, \\
& \tilde{n}_{13, t_{5}}\left(x_{3}\right)=0.5147, \tilde{n}_{23, t_{3}}\left(x_{5}\right)=0.133
\end{aligned}
$$

We can obtain the remaining combined probability based on Eq. (18)

$$
\begin{aligned}
& \tilde{n}_{H 3, t_{1}}\left(x_{1}\right)=0.34, \tilde{n}_{H 3, t_{1}}\left(x_{2}\right)=0.3832, \tilde{n}_{H 3, t_{1}}\left(x_{3}\right)=0.3606, \tilde{n}_{H 3, t_{1}}\left(x_{4}\right)=0.3391, \tilde{n}_{H 3, t_{1}}\left(x_{5}\right)=0.4087 ; \\
& \tilde{n}_{H 3, t_{2}}\left(x_{1}\right)=0.3275, \tilde{n}_{H 3, t_{2}}\left(x_{2}\right)=0.4131, \tilde{n}_{H 3, t_{2}}\left(x_{3}\right)=0.3537, \tilde{n}_{H 3, t_{2}}\left(x_{4}\right)=0.3187, \tilde{n}_{H 3, t_{2}}\left(x_{5}\right)=0.4163 ; \\
& \tilde{n}_{H 3, t_{3}}\left(x_{1}\right)=0.3349, \tilde{n}_{H 3, t_{3}}\left(x_{2}\right)=0.4024, \tilde{n}_{H 3, t_{3}}\left(x_{3}\right)=0.3905, \tilde{n}_{H 3, t_{3}}\left(x_{4}\right)=0.3486, \tilde{n}_{H 3, t_{3}}\left(x_{5}\right)=0.3522 .
\end{aligned}
$$

Step 1.3 Aggregate the evaluating values of with respect to attribute $a_{1}, a_{2}, a_{3}$ of alternative $x_{1}, x_{2}, x_{3}$ at the periods $t_{1}, t_{2}, t_{3}$ to obtain the belief distributions based on Eq. (19) as follows:

$$
\begin{aligned}
& \beta_{1, t_{1}}\left(x_{1}\right)=0.8422, \beta_{2, t_{1}}\left(x_{1}\right)=0.0978, \beta_{1, t_{1}}\left(x_{2}\right)=0.6595, \beta_{2, t_{1}}\left(x_{2}\right)=0.2305, \\
& \beta_{1, t_{1}}\left(x_{3}\right)=0.6381, \beta_{2, t_{1}}\left(x_{3}\right)=0.3019, \beta_{1, t_{1}}\left(x_{4}\right)=0.8251, \beta_{2, t_{1}}\left(x_{4}\right)=0.1349, \\
& \beta_{1, t_{1}}\left(x_{5}\right)=0.6702, \beta_{2, t_{1}}\left(x_{5}\right)=0.1198 ; \beta_{1, t_{2}}\left(x_{1}\right)=0.8611, \beta_{1, t_{2}}\left(x_{1}\right)=0.1089, \\
& \beta_{1, t_{2}}\left(x_{2}\right)=0.675, \beta_{1, t_{2}}\left(x_{2}\right)=0.135, \beta_{1, t_{2}}\left(x_{3}\right)=0.7061, \beta_{1, t_{2}}\left(x_{3}\right)=0.2539, \\
& \beta_{1, t_{2}}\left(x_{4}\right)=0.8805, \beta_{1, t_{2}}\left(x_{4}\right)=0.1195, \beta_{1, t_{2}}\left(x_{5}\right)=0.5913, \beta_{1, t_{2}}\left(x_{5}\right)=0.2187 ; \\
& \beta_{1, t_{3}}\left(x_{1}\right)=0.866, \beta_{1, t_{3}}\left(x_{1}\right)=0.074, \beta_{1, t_{3}}\left(x_{2}\right)=0.688, \beta_{1, t_{3}}\left(x_{2}\right)=0.142, \\
& \beta_{1, t_{3}}\left(x_{3}\right)=0.6853, \beta_{1, t_{3}}\left(x_{3}\right)=0.1847, \beta_{1, t_{3}}\left(x_{4}\right)=0.82, \beta_{1, t_{3}}\left(x_{4}\right)=0.1102 \\
& \beta_{1, t_{3}}\left(x_{5}\right)=0.7469, \beta_{1, t_{3}}\left(x_{5}\right)=0.1931
\end{aligned}
$$

Let the aggregated value obtained by the belief distributions $\beta_{1, t_{k}}\left(x_{i}\right), \beta_{2, t_{k}}\left(x_{i}\right)$ form the intuitionistic fuzzy values $\left(\beta_{1, t_{k}}\left(x_{i}\right), \beta_{2, t_{k}}\left(x_{i}\right)\right)$ as follows:

$$
\begin{aligned}
& \left(\beta_{1, t_{1}}\left(x_{1}\right), \beta_{2, t_{1}}\left(x_{1}\right)\right)=(0.8422,0.0978),\left(\beta_{1, t_{1}}\left(x_{2}\right), \beta_{2, t_{1}}\left(x_{2}\right)\right)=(0.6595,0.2305), \\
& \left(\beta_{1, t_{1}}\left(x_{3}\right), \beta_{2, t_{1}}\left(x_{3}\right)\right)=(0.6381,0.3019),\left(\beta_{1, t_{1}}\left(x_{4}\right), \beta_{2, t_{1}}\left(x_{4}\right)\right)=(0.8251,0.1349), \\
& \left(\beta_{1, t_{1}}\left(x_{5}\right), \beta_{2, t_{1}}\left(x_{5}\right)\right)=(0.6702,0.1198) ;\left(\beta_{1, t_{2}}\left(x_{1}\right), \beta_{1, t_{2}}\left(x_{1}\right)\right)=(0.8611,0.1089), \\
& \left(\beta_{1, t_{2}}\left(x_{2}\right), \beta_{1, t_{2}}\left(x_{2}\right)\right)=(0.675,0.135),\left(\beta_{1, t_{2}}\left(x_{3}\right), \beta_{1, t_{2}}\left(x_{3}\right)\right)=(0.7061,0.2539), \\
& \left(\beta_{1, t_{2}}\left(x_{4}\right), \beta_{1, t_{2}}\left(x_{4}\right)\right)=(0.8805,0.1195),\left(\beta_{1, t_{2}}\left(x_{5}\right), \beta_{1, t_{2}}\left(x_{5}\right)\right)=(=0.5913,0.2187) ; \\
& \left(\beta_{1, t_{3}}\left(x_{1}\right), \beta_{1, t_{3}}\left(x_{1}\right)\right)=(0.866,0.074),\left(\beta_{1, t_{3}}\left(x_{2}\right), \beta_{1, t_{3}}\left(x_{2}\right)\right)=(0.688,0.142), \\
& \left(\beta_{1, t_{3}}\left(x_{3}\right), \beta_{1, t_{3}}\left(x_{3}\right)\right)=(0.6853,0.1847),\left(\beta_{1, t_{3}}\left(x_{4}\right), \beta_{1, t_{3}}\left(x_{4}\right)\right)=(0.82,0.1102) \\
& \left(\beta_{1, t_{3}}\left(x_{5}\right), \beta_{1, t_{3}}\left(x_{5}\right)\right)=(0.7469,0.1931)
\end{aligned}
$$


Step 2. Based on Step 1, construct the aggregation decision making matrix $Q$ as follows

$$
\begin{aligned}
Q & =\left(\beta_{1, t_{k}}\left(x_{i}\right), \beta_{2, t_{k}}\left(x_{i}\right)\right)_{3 \times 3} \\
& =\left(\begin{array}{lll}
\left(\beta_{1, t_{1}}\left(x_{1}\right), \beta_{2, t_{1}}\left(x_{1}\right)\right) & \left(\beta_{1, t_{2}}\left(x_{1}\right), \beta_{2, t_{2}}\left(x_{1}\right)\right) & \left(\beta_{1, t_{3}}\left(x_{1}\right), \beta_{2, t_{3}}\left(x_{1}\right)\right) \\
\left(\beta_{1, t_{1}}\left(x_{2}\right), \beta_{2, t_{1}}\left(x_{2}\right)\right) & \left(\beta_{1, t_{2}}\left(x_{2}\right), \beta_{2, t_{2}}\left(x_{2}\right)\right) & \left(\beta_{1, t_{3}}\left(x_{2}\right), \beta_{2, t_{3}}\left(x_{2}\right)\right) \\
\left(\beta_{1, t_{1}}\left(x_{3}\right), \beta_{2, t_{1}}\left(x_{3}\right)\right) & \left(\beta_{1, t_{2}}\left(x_{3}\right), \beta_{2, t_{2}}\left(x_{3}\right)\right) & \left(\beta_{1, t_{3}}\left(x_{3}\right), \beta_{2, t_{3}}\left(x_{3}\right)\right) \\
\left(\beta_{1, t_{1}}\left(x_{4}\right), \beta_{2, t_{1}}\left(x_{4}\right)\right) & \left(\beta_{1, t_{2}}\left(x_{4}\right), \beta_{2, t_{2}}\left(x_{4}\right)\right) & \left(\beta_{1, t_{3}}\left(x_{4}\right), \beta_{2, t_{3}}\left(x_{4}\right)\right) \\
\left(\beta_{1, t_{1}}\left(x_{5}\right), \beta_{2, t_{1}}\left(x_{5}\right)\right) & \left(\beta_{1, t_{2}}\left(x_{5}\right), \beta_{2, t_{2}}\left(x_{5}\right)\right) & \left(\beta_{1, t_{3}}\left(x_{5}\right), \beta_{2, t_{3}}\left(x_{5}\right)\right)
\end{array}\right) \\
& =\left(\begin{array}{lll}
(0.8422,0.0978) & (0.8611,0.1089) & (0.866,0.074) \\
(0.6595,0.2305) & (0.675,0.135) & (0.688,0.142) \\
(0.6381,0.3019) & (0.7061,0.2536) & (0.6853,0.1847) \\
(0.8251,0.1349) & (0.8805,0.1195) & (0.8198,0.1102) \\
(0.6702,0.1198) & (0.5913,0.2187) & (0.7469,0.1931)
\end{array}\right)
\end{aligned}
$$

Step 2.1. Based on Step 2 and Eq. (21), we can obtain the basic probability mass matrix

$$
Q=\left(\begin{array}{ccc}
(0.1684,0.0196) & (0.2583,0.0327) & (0.433,0.037) \\
(0.132,0.04611) & (0.2025,0.0405) & (0.344,0.071) \\
(0.1276,0.06037) & (0.2118,0.0762) & (0.3426,0.0924) \\
(0.165,0.027) & (0.26416,0.0358) & (0.41,0.0551) \\
(0.134,0.024) & (0.1774,0.0656) & (0.3735,0.0965)
\end{array}\right)
$$

Step 2.2. Based on Eq. (23) and Eq. (24), we can calculate the combined probability mass $\tilde{n}_{q, t_{k}}\left(x_{i}\right)$ and $\tilde{n}_{H, t_{k}}\left(x_{i}\right)$ w.r.t. alternative $x_{i}$ at the period $t_{k}$ as follows:

$$
\begin{aligned}
& n_{1,3}\left(x_{1}\right)=0.635, n_{2,3}\left(x_{1}\right)=0.0465, n_{1,3}\left(x_{2}\right)=0.5171, n_{2,3}\left(x_{2}\right)=0.0959, n_{1,3}\left(x_{3}\right)=0.5051, \\
& n_{2,3}\left(x_{3}\right)=0.1372, n_{1,3}\left(x_{4}\right)=0.6169, n_{2,3}\left(x_{4}\right)=0.0634, n_{1,3}\left(x_{5}\right)=0.5214, n_{2,3}\left(x_{5}\right)=0.1163, \\
& n_{H, 3}\left(x_{1}\right)=0.3185, n_{H, 3}\left(x_{2}\right)=0.3871, n_{H, 3}\left(x_{3}\right)=0.3576, n_{H, 3}\left(x_{4}\right)=0.3197, n_{H, 3}\left(x_{5}\right)=0.3622 .
\end{aligned}
$$

Step 2.3. Aggregate the evaluating values of decision makers with respect to alternative $x_{i}$ to obtain the belief distributions based on Eq. (25)

$$
\begin{aligned}
& \beta_{1}\left(x_{1}\right)=0.8842, \beta_{2}\left(x_{1}\right)=0.0648, \beta_{1}\left(x_{2}\right)=0.7053, \beta_{2}\left(x_{2}\right)=0.1307, \beta_{1}\left(x_{3}\right)=0.7163 \\
& \beta_{2}\left(x_{3}\right)=0.1947, \beta_{1}\left(x_{4}\right)=0.8679, \beta_{2}\left(x_{4}\right)=0.0891, \beta_{1}\left(x_{5}\right)=0.7121, \beta_{2}\left(x_{5}\right)=0.1589
\end{aligned}
$$

Let the aggregated value obtained by the belief distributions $\beta_{1}\left(x_{i}\right), \beta_{2}\left(x_{i}\right)$ form the intuitionistic fuzzy values $\left(\beta_{1}\left(x_{i}\right), \beta_{2}\left(x_{i}\right)\right)$ as follows:

$$
\begin{aligned}
& \left(\beta_{1}\left(x_{1}\right), \beta_{2}\left(x_{1}\right)\right)=(0.8842,0.0648),\left(\beta_{1}\left(x_{2}\right), \beta_{2}\left(x_{2}\right)\right)=(0.7053,0.1307), \\
& \left(\beta_{1}\left(x_{3}\right), \beta_{2}\left(x_{3}\right)\right)=(0.7163,0.1947),\left(\beta_{1}\left(x_{4}\right), \beta_{2}\left(x_{4}\right)\right)=(0.8679,0.0891), \\
& \left(\beta_{1}\left(x_{5}\right), \beta_{2}\left(x_{5}\right)\right)=(0.7121,0.1589) .
\end{aligned}
$$

Step 3 Calculate the scores of IFN $\left(\beta_{1}\left(x_{i}\right), \beta_{2}\left(x_{i}\right)\right)$ obtained by the aggregation result of Step 3. Let $\alpha_{i}=\left(\beta_{1}\left(x_{i}\right), \beta_{2}\left(x_{i}\right)\right)(i=1,2,3,4,5)$

Step 3.1 According to Eq. (2), calculate $H_{M}\left(\alpha_{i}\right)(i=1,2,3,4,5)$ as follows:

$H_{M}\left(\alpha_{1}\right)=0.5665, H_{M}\left(\alpha_{2}\right)=0.2634, H_{M}\left(\alpha_{3}\right)=0.2085, H_{M}\left(\alpha_{4}\right)=0.5, H_{M}\left(\alpha_{5}\right)=0.2391$.

Step 3.2 Calculate the score of $\alpha_{i}$ and denote as $S\left(\alpha_{i}\right)(i=1,2,3)$ according to Eq. (3): 
Step 4 Determine the ranking of alternatives according to Step 3. We can obtained the preference order of alternatives as $x_{1}>x_{4}>x_{2}>x_{5}>x_{3}$, that is, $x_{1}$ is the desirable one.

(2) Method II: We utilize the MDIFWG which is presented in Section 4.2.2.

Step 1. Utilize the MDIFWG operator to aggregate all the intuitionistic fuzzy decision matrices $D_{t_{k}}=\left(\alpha_{i j, t_{k}}\right)_{5 \times 3}(k=1,2,3)$ into a complex intuitionistic fuzzy decision matrix $D=\left(\alpha_{i j}\right)_{5 \times 3}$ as follows, where $\alpha_{i j}=\left(\mu_{i j}, v_{i j}\right)$ is an IFN obtained by Eq. (30).

$$
D=\left(\begin{array}{ccc}
(0.801,0.199) & (0.8769,0.1231) & (0.8466,0.1534) \\
(0.8466,0.1534) & (0.5723,0.1815) & (0.5898,0.237) \\
(0.4523,0.5477) & (0.7551,0.2449) & (0.7186,0.2814) \\
(0.8586,0.1414) & (0.7842,0.2158) & (0.8403,0.1597) \\
(0.5723,0.2545) & (0.7538,0.2462), & (0.6378,0.1998)
\end{array}\right)
$$

Step 2-3. Calculate the distance between the alternative $x_{i}$ and the IFPIS $\alpha^{+}$and the distance between the alternative $x_{i}$ and the IFNIS $\alpha^{-}$, respectively:

$$
\begin{aligned}
& d\left(x_{1}, \alpha^{+}\right)=0.1549, d\left(x_{2}, \alpha^{+}\right)=0.3402, d\left(x_{3}, \alpha^{+}\right)=0.3467, d\left(x_{4}, \alpha^{+}\right)=0.1766, d\left(x_{5}, \alpha^{+}\right)=0.3355 ; \\
& d\left(x_{1}, \alpha^{-}\right)=0.8451, d\left(x_{2}, \alpha^{-}\right)=0.8103, d\left(x_{3}, \alpha^{-}\right)=0.6533, d\left(x_{4}, \alpha^{-}\right)=0.8234, d\left(x_{5}, \alpha^{-}\right)=0.7652 .
\end{aligned}
$$

Step 4. Calculate the closeness coefficient of each alternative

$$
c\left(x_{1}\right)=0.8451, c\left(x_{2}\right)=0.7043, c\left(x_{3}\right)=0.6533, c\left(x_{4}\right)=0.8234, c\left(x_{5}\right)=0.6952 .
$$

Step 5. According to Step 4, we can obtain the preference order as $x_{1}>x_{4}>x_{2}>x_{5}>x_{3}$, which coincides with the order obtained by using Method I.

Table 4 shows a comparison of the preference order of the alternatives for different methods for Example 3.

Table 4: A comparison of preference order for different methos

\begin{tabular}{cl}
\hline methods & preference order \\
\hline DIFWA[39] & $x_{1}>x_{4}>x_{2}>x_{5}>x_{3}$ \\
DIFWG[32] & $x_{1}>x_{4}>x_{2}>x_{5}>x_{3}$ \\
DIFWG ${ }^{[15]}$ & $x_{1}>x_{4}>x_{2}>x_{5}>x_{3}$ \\
Extended VIKOR based on DIFWG[26] & $x_{1}>x_{4}>x_{5}>x_{2}>x_{3}$ \\
The proposed method based MDIFWG & $x_{1}>x_{4}>x_{2}>x_{5}>x_{3}$ \\
The proposed method based ER algorithm & $x_{1}>x_{4}>x_{2}>x_{5}>x_{3}$ \\
\hline
\end{tabular}

It follows from the Table 4 that the preference order of alternatives obtained by our proposed method are the same with the preference order obtained by Xu's [39], Gumus's [15] and Wei's [32] methods. It is also shown that our proposed methods based on ER algorithm and MDIFWG operators are valid.

Now, the following two examples will be used to show the our proposed methods can overcome effectively the Drawbacks A, B and C listed in Section 3.

Example 4. Considering Example 2, we illustrate how this example can be solved by using the proposed two methods in Section 4. 
(1) Method I. Utilizing the ER algorithm. The specific steps are detailed as follows:

Step 1. Based on the decision matrices $D_{t_{1}}, D_{t_{2}}$ and $D_{t_{3}}$, as well as the weights of attributes and Eq. (15), Eq. (16), we can obtain the basic probability mass $P_{t_{1}}, P_{t_{2}}$ and $P_{t_{3}}$ :

$$
\begin{gathered}
P_{t_{1}}=\left(\begin{array}{ccc}
(0.0833,0.2333) & (0.1667,0.0667) & (0.2333,0.0667) \\
(0.1667,0.0667) & (0.1333,0.16665) & (0.1333,0.0333) \\
(0.1333,0.1) & (0.1667,0.1000) & (0.2,0.1)
\end{array}\right) \\
P_{t_{2}}=\left(\begin{array}{ccc}
(0.2,0.1) & (0.1333,0.0333) & (0.20 .0333) \\
(0.2333,0.0333) & (0.0833,0.2333) & (0.1667,0.1) \\
(0.1667,0.0667) & (0.2333,0.0667) & (0.1333,0.1667)
\end{array}\right) \\
P_{t_{3}}=\left(\begin{array}{ccc}
(0.1333,0.1667) & (0.2333,0.1) & (0.1661,0.1) \\
(0.2,0.1) & (0.2,0.1) & (0.2333,0.0667) \\
(0.2333,0.0333) & (0.2,0.0333) & (0.08333,0.2333)
\end{array}\right)
\end{gathered}
$$

Step 1.2. We can obtain the combined probability based on Eq. (17):

$$
\begin{aligned}
& \tilde{n}_{13, t_{1}}\left(x_{1}\right)=0.3396, \tilde{n}_{23, t_{1}}\left(x_{1}\right)=0.2467, \tilde{n}_{13, t_{1}}\left(x_{2}\right)=0.3273, \tilde{n}_{23, t_{1}}\left(x_{2}\right)=0.1920, \\
& \tilde{n}_{13, t_{1}}\left(x_{2}\right)=0.3673, \tilde{n}_{23, t_{1}}\left(x_{3}\right)=0.2017, \tilde{n}_{13, t_{2}}\left(x_{1}\right)=0.4152, \tilde{n}_{13, t_{2}}\left(x_{1}\right)=0.1133, \\
& \tilde{n}_{13, t_{2}}\left(x_{2}\right)=0.3384, \tilde{n}_{13, t_{2}}\left(x_{2}\right)=0.2465, \tilde{n}_{13, t_{2}}\left(x_{3}\right)=0.3874, \tilde{n}_{13, t_{2}}\left(x_{3}\right)=0.1969, \\
& \tilde{n}_{13, t_{3}}\left(x_{1}\right)=0.3754, \tilde{n}_{13, t_{3}}\left(x_{1}\right)=0.2385, \tilde{n}_{13, t_{3}}\left(x_{2}\right)=0.4570, \tilde{n}_{13, t_{3}}\left(x_{2}\right)=0.1634, \\
& \tilde{n}_{13, t_{3}}\left(x_{3}\right)=0.3714, \tilde{n}_{13, t_{3}}\left(x_{3}\right)=0.1990 .
\end{aligned}
$$

We can then obtain the remaining combined probability based on Eq. (18):

$$
\begin{aligned}
& \tilde{n}_{H 3, t_{1}}\left(x_{1}\right)=0.4136, \tilde{n}_{H 3, t_{1}}\left(x_{2}\right)=0.4806, \tilde{n}_{H 3, t_{1}}\left(x_{3}\right)=0.431 ; \\
& \tilde{n}_{H 3, t_{2}}\left(x_{1}\right)=0.4716, \tilde{n}_{H 3, t_{2}}\left(x_{2}\right)=0.4152, \tilde{n}_{H 3, t_{2}}\left(x_{3}\right)=0.4156 ; \\
& \tilde{n}_{H 3, t_{3}}\left(x_{1}\right)=0.3861, \tilde{n}_{H 3, t_{3}}\left(x_{2}\right)=0.3796, \tilde{n}_{H 3, t_{3}}\left(x_{3}\right)=0.4296 .
\end{aligned}
$$

Step 1.3. Aggregate the evaluating values with respect to attribute $a_{1}, a_{2}, a_{3}$ of alternative $x_{1}, x_{2}, x_{3}$ at the periods $t_{1}, t_{2}, t_{3}$ to obtain the belief distributions based on Eq.(19):

$$
\begin{aligned}
& \beta_{1, t_{1}}\left(x_{1}\right)=0.4922, \beta_{2, t_{1}}\left(x_{1}\right)=0.3578, \beta_{1, t_{1}}\left(x_{2}\right)=0.4412, \beta_{2, t_{1}}\left(x_{2}\right)=0.2588, \\
& \beta_{1, t_{1}}\left(x_{3}\right)=0.5164, \beta_{2, t_{1}}\left(x_{3}\right)=0.2836, \beta_{1, t_{2}}\left(x_{1}\right)=0.55, \beta_{1, t_{2}}\left(x_{1}\right)=0.1501, \\
& \beta_{1, t_{2}}\left(x_{2}\right)=0.4918, \beta_{1, t_{2}}\left(x_{2}\right)=0.3582, \beta_{1, t_{2}}\left(x_{3}\right)=0.5525, \beta_{1, t_{2}}\left(x_{3}\right)=0.2808, \\
& \beta_{1, t_{3}}\left(x_{1}\right)=0.5503, \beta_{1, t_{3}}\left(x_{1}\right)=0.3497, \beta_{1, t_{3}}\left(x_{2}\right)=0.663, \beta_{1, t_{3}}\left(x_{2}\right)=0.237, \\
& \beta_{1, t_{3}}\left(x_{3}\right)=0.5317, \beta_{1, t_{3}}\left(x_{3}\right)=0.285 .
\end{aligned}
$$

Let the aggregated values be obtained by the above belief distributions $\beta_{1, t_{k}}\left(x_{i}\right), \beta_{2, t_{k}}\left(x_{i}\right)$ form the intuitionistic fuzzy values $\left(\beta_{1, t_{k}}\left(x_{i}\right), \beta_{2, t_{k}}\left(x_{i}\right)\right)$ as follows:

$$
\begin{aligned}
& \left(\beta_{1, t_{1}}\left(x_{1}\right), \beta_{2, t_{1}}\left(x_{1}\right)\right)=(0.4922,0.3578),\left(\beta_{1, t_{2}}\left(x_{1}\right), \beta_{1, t_{2}}\left(x_{1}\right)\right)=(0.55,0.1501), \\
& \left(\beta_{1, t_{3}}\left(x_{1}\right), \beta_{1, t_{3}}\left(x_{1}\right)\right)=(0.5503,0.3497),\left(\beta_{1, t_{1}}\left(x_{2}\right), \beta_{2, t_{1}}\left(x_{2}\right)\right)=(0.4412,0.2588), \\
& \left(\beta_{1, t_{2}}\left(x_{2}\right), \beta_{1, t_{2}}\left(x_{2}\right)\right)=(0.4918,0.3582),\left(\beta_{1, t_{3}}\left(x_{2}\right), \beta_{1, t_{3}}\left(x_{2}\right)\right)=(0.663,0.237), \\
& \left(\beta_{1, t_{1}}\left(x_{3}\right), \beta_{2, t_{1}}\left(x_{3}\right)\right)=(0.5164,0.2836),\left(\beta_{1, t_{2}}\left(x_{3}\right), \beta_{1, t_{2}}\left(x_{3}\right)\right)=(0.5525,0.2808), \\
& \left(\beta_{1, t_{3}}\left(x_{3}\right), \beta_{1, t_{3}}\left(x_{3}\right)\right)=(0.5317,0.285) .
\end{aligned}
$$


Step 2. Based on Step 1, construct the aggregation decision making matrix $Q$ as follows:

$$
\begin{aligned}
Q & =\left(\beta_{1, t_{k}}\left(x_{i}\right), \beta_{2, t_{k}}\left(x_{i}\right)\right)_{3 \times 3} \\
& =\left(\begin{array}{lll}
\left(\beta_{1, t_{1}}\left(x_{1}\right), \beta_{2, t_{1}}\left(x_{1}\right)\right) & \left(\beta_{1, t_{2}}\left(x_{1}\right), \beta_{2, t_{2}}\left(x_{1}\right)\right) & \left(\beta_{1, t_{3}}\left(x_{1}\right), \beta_{2, t_{3}}\left(x_{1}\right)\right) \\
\left(\beta_{1, t_{1}}\left(x_{2}\right), \beta_{2, t_{1}}\left(x_{2}\right)\right) & \left(\beta_{1, t_{2}}\left(x_{2}\right), \beta_{2, t_{2}}\left(x_{2}\right)\right) & \left(\beta_{1, t_{3}}\left(x_{2}\right), \beta_{2, t_{3}}\left(x_{2}\right)\right) \\
\left(\beta_{1, t_{1}}\left(x_{3}\right), \beta_{2, t_{1}}\left(x_{3}\right)\right) & \left(\beta_{1, t_{2}}\left(x_{3}\right), \beta_{2, t_{2}}\left(x_{3}\right)\right) & \left(\beta_{1, t_{3}}\left(x_{3}\right), \beta_{2, t_{3}}\left(x_{3}\right)\right)
\end{array}\right) \\
& =\left(\begin{array}{lll}
(0.4922,0.3578) & (0.55,0.1501) & (0.5503,0.3497) \\
(0.4412,0.2588) & (0.4918,0.3582) & (0.663,0.237) \\
(0.5164,0.2836) & (0.5525,0.2808) & (0.5317,0.285)
\end{array}\right)
\end{aligned}
$$

Step 2.1. Based on Step 2 and Eq. (21), we can obtain the basic probability mass matrix:

$$
Q=\left(\begin{array}{ccc}
(0.164,0.119) & (0.1833,0.05) & (0.1834,0.1166) \\
(0.1471,0.0863) & (0.1639,0.1194) & (0.221,0.07901) \\
(0.1721,0.09453) & (0.18415,0.0936) & (0.17723,0.095)
\end{array}\right)
$$

Step 2.2. Based on Eq. (23) and Eq. (24), we can calculate the combined probability mass $\tilde{n}_{q, t_{k}}\left(x_{i}\right)$ and $\tilde{n}_{C, t_{k}}\left(x_{i}\right)$ w.r.t. alternative $x_{i}$ at period $t_{k}$ as follows:

$$
\begin{aligned}
& n_{1,3}\left(x_{1}\right)=0.3887, n_{2,3}\left(x_{1}\right)=0.1895, n_{1,3}\left(x_{2}\right)=0.3908, n_{2,3}\left(x_{2}\right)=0.1874, \\
& n_{1,3}\left(x_{3}\right)=0.3913, n_{2,3}\left(x_{3}\right)=0.1865 \\
& n_{H, 3}\left(x_{1}\right)=0.4218, n_{H, 3}\left(x_{2}\right)=0.4218, n_{H, 3}\left(x_{3}\right)=0.4222
\end{aligned}
$$

Step 2.3. Aggregate the evaluating values of decision makers with respect to alternative $x_{i}$ to obtain the following belief distributions based on Eq. (25):

$$
\begin{aligned}
& \beta_{1}\left(x_{1}\right)=0.549, \beta_{2}\left(x_{1}\right)=0.2677 \\
& \beta_{1}\left(x_{2}\right)=0.552, \beta_{2}\left(x_{2}\right)=0.2647 \\
& \beta_{1}\left(x_{3}\right)=0.5531, \beta_{2}\left(x_{3}\right)=0.2636
\end{aligned}
$$

Let the aggregated values obtained by the above belief distributions $\beta_{1}\left(x_{i}\right), \beta_{2}\left(x_{i}\right)$ form the intuitionistic fuzzy values $\left(\beta_{1}\left(x_{i}\right), \beta_{2}\left(x_{i}\right)\right)$ as follows:

$$
\begin{aligned}
& \left(\beta_{1}\left(x_{1}\right), \beta_{2}\left(x_{1}\right)\right)=(0.549,0.2677) ; \\
& \left(\beta_{1}\left(x_{2}\right), \beta_{2}\left(x_{2}\right)\right)=(0.552,0.2647) ; \\
& \left.\beta_{1}\left(x_{3}\right), \beta_{2}\left(x_{3}\right)\right)=(0.5531,0.2636) .
\end{aligned}
$$

3.

Step 3. Calculate the scores of IFN $\left(\beta_{1}\left(x_{i}\right), \beta_{2}\left(x_{i}\right)\right)$ obtained by the aggregation result of Step

Let $\alpha_{i}=\left(\beta_{1}\left(x_{i}\right), \beta_{2}\left(x_{i}\right)\right)(i=1,2, m)$.

Step 3.1. According to Eq. (2), calculate $H_{M}\left(\alpha_{i}\right)(i=1,2,3)$ and shown as follows:

$H_{M}\left(\alpha_{1}\right)=0.06, H_{M}\left(\alpha_{1}\right)=0.06266, H_{M}\left(\alpha_{1}\right)=0.06367$.

Step 3.2. Calculate the score of $\alpha_{i}$ and denote $S\left(\alpha_{i}\right)(i=1,2,3)$ according to Eq. (3): $S\left(\alpha_{1}\right)=0.2923, S\left(\alpha_{2}\right)=0.2988, S\left(\alpha_{3}\right)=0.3013$.

Step 4. Determine the ranking of alternatives according to Step 3. We can obtain the preference order of alternatives is $x_{3}>x_{2}>x_{1}$, that is, $x_{3}$ is the desirable one. 
We can see from Exa. 2 that the DIF-MADM methods proposed by Xu [39], Gumus [15] and Wei [32] can not distinguish the preference order of alternatives $x_{1}, x_{2}, x_{3}$. However, we can see from above Method I that our DIF-MADM method based on ER algorithm can distinguish the preference order of alternatives $x_{1}, x_{2}, x_{3}$. It is also shown that our method based on ER algorithm can overcome the Drawback C. That is, Drawback C is not the drawback anymore in this new method based on ER algorithm.

(2) Method II. We utilized the MDIFWG which is presented in Section 4.2.2. for Example 2.

Step 1. Utilize the MDIFWG operator to aggregate all the intuitionistic fuzzy decision matrices $D_{t_{k}}=\left(\alpha_{i j, t_{k}}\right)_{3 \times 3}(k=1,2,3)$ into a complex intuitionistic fuzzy decision matrix $D=\left(\alpha_{i j}\right)_{3 \times 3}$ as follows, where $\alpha_{i j}=\left(\mu_{i j}, v_{i j}\right)$ is an IFN obtained by Eq. (30).

$$
D=\left(\begin{array}{ccc}
(0.3926,0.5279) & (0.796,0.204) & (0.614,0.204) \\
(0.614,0.204) & (0.3926,0.5279) & (0.5802,0.204) \\
(0.5336,0.204) & (0.614,0.204) & (0.3926,0.5279)
\end{array}\right)
$$

Step 2-3. Calculate the distance between the alternative $x_{i}$ and the IFPIS $\alpha^{+}$and the distance between the alternative $x_{i}$ and the IFNIS $\alpha^{-}$, respectively:

$$
\begin{aligned}
& d\left(x_{1}, \alpha^{+}\right)=0.5768, d\left(x_{2}, \alpha^{+}\right)=0.5191, d\left(x_{3}, \alpha^{+}\right)=0.5048 \\
& d\left(x_{1}, \alpha^{-}\right)=d\left(x_{2}, \alpha^{-}\right)=d\left(x_{3}, \alpha^{-}\right)=0.3309
\end{aligned}
$$

Step 4. Calculate the closeness coefficient of each alternative

$$
c\left(x_{1}\right)=0.3646, c\left(x_{2}\right)=0.3893, c\left(x_{3}\right)=0.396 .
$$

Step 5. According to Step 4, we can obtain the preference order is $x_{3}>x_{2}>x_{1}$, which coincided with the order obtained by using Method I as detailed above.

Table 5 shows a comparison of the preference order of the alternatives for different methods for Example 4.

Table 5: A comparison of preference order for different methods for Example 3

\begin{tabular}{cc}
\hline Methods & Preference order \\
\hline DIFWA[39] & $x_{1}=x_{2}=x_{3}$ \\
DIFWG[32] & $x_{1}=x_{2}=x_{3}$ \\
DIFWG $[15]$ & $x_{1}=x_{2}=x_{3}$ \\
Extended VIKOR based on DIFWG[26] & $x_{1}=x_{2}=x_{3}$ \\
The proposed method based on MDIFWG & $x_{1}<x_{2}<x_{3}$ \\
The proposed method based on the ER algorithm & $x_{1}<x_{2}<x_{3}$ \\
\hline
\end{tabular}

We can see from Table 4 that the DIF-MADM methods proposed by Xu [39], Gumus [15] and Wei [32] can not distinguish the preference order of alternatives $x_{1}, x_{2}, x_{3}$. The same problem is also obtained by extend VIKOR method based on DIFWG, the root of this problem is the related aggregation operators (or the definition of operation of dynamic intuitionistic fuzzy numbers). However, we can see from above Table 5 that our DIF-MADM methods based on ER algorithm 
and MDIFWG operator can distinguish the preference order of alternatives $x_{1}, x_{2}, x_{3}$. It is also shown that our methods based on ER algorithm and MDIFWG operator can overcome effectively the Drawback C.

The following example can show the proposed methods can overcome the drawbacks A and B of existing methods analysed in Section 3.

Example 5. A company wants to invest in one of renewable energy sources, Geothermal, solar, biomass among renewable energy sources have been determined as alternatives. The company has determined three criteria for the evaluation of renewable energy resources: risk factor, the ${ }_{430}$ growth rate in the sector, payback reliability. The company thinks that these evaluations need to be done in a dynamic process due to the increasing energy demand, environmental awareness and government support for energy projects in recent three years. The three alternative $x_{i}(i=1,2,3)$ : (1) $x_{1}$ is Geothermal; (2) $x_{2}$ is solar; (3) $x_{3}$ is biomass. The investment company must take a decision according to the following three attributes: (1) $A_{1}$ is risk factor; (2) $A_{2}$ is the growth

435 rate in the sector; (3) $A_{3}$ is the payback reliability. The three possible alternatives $x_{i}(i=1,2,3)$ are to be evaluated using the intuitionistic fuzzy information by the decision maker under the above four attributes at the periods $t_{k}(k=1,2,3)$, as listed in the following matrix, shown as Table 6. Let $\lambda(t)=\left(\frac{1}{3}, \frac{1}{3}, \frac{1}{3}\right)$ be weight vector of the periods $t_{k}(k=1,2,3)$, and $w=\left(\frac{1}{3}, \frac{1}{3}, \frac{1}{3}\right)$ be weight vector of the attributes $A_{j}(j=1,2,3)$.

Table 6: Individual IF decision matrix $D_{t_{k}}(k=1,2,3)$

\begin{tabular}{ccccc}
\hline years & & $A_{1}$ & $A_{2}$ & $A_{3}$ \\
\hline \multirow{4}{*}{$t_{1}$} & $x_{1}$ & $(0.6,0.1)$ & $(0.5,0.2)$ & $(0.7,0.2)$ \\
& $x_{2}$ & $(0,0.1)$ & $(0.4,0.5)$ & $(0.4,0.1)$ \\
& $x_{3}$ & $(0.4,0.3)$ & $(0.5,0.1)$ & $(0.6,0.3)$ \\
\hline \multirow{4}{*}{$t_{2}$} & $x_{1}$ & $(0.6,0.3)$ & $(0.7,0.1)$ & $(0.6,0.1)$ \\
& $x_{2}$ & $(0.7,0.2)$ & $(0.1,0.3)$ & $(0.5,0.3)$ \\
& $x_{3}$ & $(0.5,0.2)$ & $(0.7,0.2)$ & $(0,0.1)$ \\
\hline \multirow{3}{*}{$t_{3}$} & $x_{1}$ & $(0.4,0.5)$ & $(0.6,0.3)$ & $(0.5,0.4)$ \\
& $x_{2}$ & $(0.5,0.4)$ & $(0.6,0.1)$ & $(0.7,0.2)$ \\
& $x_{3}$ & $(0.7,0.1)$ & $(0.5,0.4)$ & $(0.1,0.5)$ \\
\hline
\end{tabular}

The results obtained by the proposed methods based on Method I and Method II are listed in Table 7. The details of process are the same as the ones in Example 3 and Example 4, so skipped. Whilst, Table 7 also shows the comparisons with some existing methods.

We can see from Table 7 that the DIF-MADM methods Gumus [15] and Wei [32] can not distinguish the preference order of alternatives $x_{2}, x_{3}$. The reason is that there is only one mem-

445 bership degree of IFNs is equal to 0 , the aggregation membership degree of IFNs is 0 even if the membership degrees of $n-1$ IFNs are not 0 , which leads to inappropriate preference order of alternatives in this situation. However, we can see from above Table 7 that our DIF-MADM methods based on ER algorithm and MDIFWG operator can distinguish the preference order of alternatives $x_{1}, x_{2}, x_{3}$. It is also shown that our methods based on ER algorithm and MDIFWG 450 operator can overcome effectively the Drawback B.

In Exa 5., if $\mu_{\alpha\left(t_{1}\right)}\left(x_{2}\right)=1$ at the period $t_{1}, \mu_{\alpha\left(t_{2}\right)}\left(x_{2}\right)=\mu_{\alpha\left(t_{3}\right)}\left(x_{2}\right)=0$, the modified decision matrix is shown as follows: 
Table 7: A comparison of preference order for different methods for Example 5

\begin{tabular}{cc}
\hline Methods & Preference order \\
\hline DIFWA[39] & $x_{1}>x_{3}>x_{2}$ \\
DIFWG[32] & $x_{2}=x_{3}>x_{1}$ \\
DIFWG ${ }^{[15]}$ & $x_{2}=x_{3}>x_{1}$ \\
The proposed method based MDIFWG & $x_{1}>x_{3}>x_{2}$ \\
The proposed method based ER algorithm & $x_{1}>x_{3}>x_{2}$ \\
\hline
\end{tabular}

Table 8: Modified individual IF decision matrix $D_{t_{k}}(k=1,2,3)$

\begin{tabular}{ccccc}
\hline years & & $A_{1}$ & $A_{2}$ & $A_{3}$ \\
\hline \multirow{4}{*}{$t_{1}$} & $x_{1}$ & $(0.2,0.6)$ & $(0.5,0.2)$ & $(0.7,0.2)$ \\
& $x_{2}$ & $(1,0)$ & $(0.4,0.5)$ & $(0.4,0.1)$ \\
& $x_{3}$ & $(0.4,0.3)$ & $(0.5,0.1)$ & $(0.6,0.3)$ \\
\hline \multirow{4}{*}{$t_{2}$} & $x_{1}$ & $(0.4,0.3)$ & $(0.7,0.1)$ & $(0.6,0.1)$ \\
& $x_{2}$ & $(0,0.2)$ & $(0.1,0.3)$ & $(0.5,0.3)$ \\
& $x_{3}$ & $(0.5,0.2)$ & $(0.7,0.2)$ & $(0,0.1)$ \\
\hline \multirow{3}{*}{$t_{3}$} & $x_{1}$ & $(0.2,0.5)$ & $(0.6,0.3)$ & $(0.5,0.4)$ \\
& $x_{2}$ & $(0,0.4)$ & $(0.6,0.1)$ & $(0.7,0.2)$ \\
& $x_{3}$ & $(0.5,0.4)$ & $(0.5,0.4)$ & $(0.1,0.5)$ \\
\hline
\end{tabular}

The results obtained by the proposed methods based on Method I is $x_{2}>x_{1}>x_{3}$. The preference order of alternatives is the same with order obtained by Xu's method based on DIFWA

455 [39]. However, the preference order of alternatives obtained by Xu's method [39] will be the same no matter how the non-membership degrees of $A_{1}$ regarding on $x_{2}$ change at period $t_{2}, t_{3}$, this situation is shown in Fig .1. Obviously, it is unreasonable.

As far as our proposed method I is concerned, the preference order will be changed with the non-membership degree of $A_{1}$ regarding on $x_{2}$ change. For example, when the non-membership degrees of $A_{1}$ regarding on $x_{2}$ change at period $t_{2}, t_{3}$, the preference order of alternatives obtained by our proposed method I is shown in Fig. 2.

We can see from the above analysis, Fig. 1 and Fig. 2 that our proposed method I can overcome the Drawback A.

\subsection{Sensitivity analysis}

Baird [6] pointed out that sensitivity analysis (SA) is the investigation of some potential changes and errors of rating values and their impact on the final ranking order. In this subsection, we conduct some sensitivity analyses to analyze the impact of changing the membership and non-membership degrees of the rating values on the alternatives ranking order based on Method I (DIF-MADM based on the ER algorithm).

$470 \quad$ For the original membership and non-membership degrees $\alpha_{t_{k}}=\left(\mu_{i j, t_{k}}, v_{i j, t_{k}}\right)$, because the sum of membership degree and the non-membership degree of a intuitionistic number is not more than 1 , so we can assume it is updated as $\left(\mu_{i j, t_{k}}+\Delta_{i j, t_{k}}, v_{i j, t_{k}}-\Delta_{i j, t_{k}}\right)$, where $\mu_{i j, t_{k}}+\Delta_{i j, t_{k}}, v_{i j, t_{k}}-\Delta_{i j, t_{k}} \in$ 


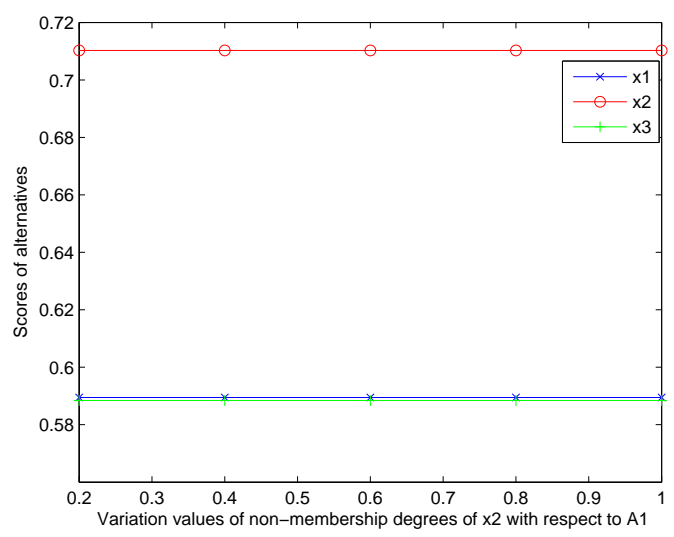

Figure 1: Ranking order sensitivity to the non-membership degrees of $x_{2}$ with respect to the first attribute $A_{1}$ by Xu's Method [39]

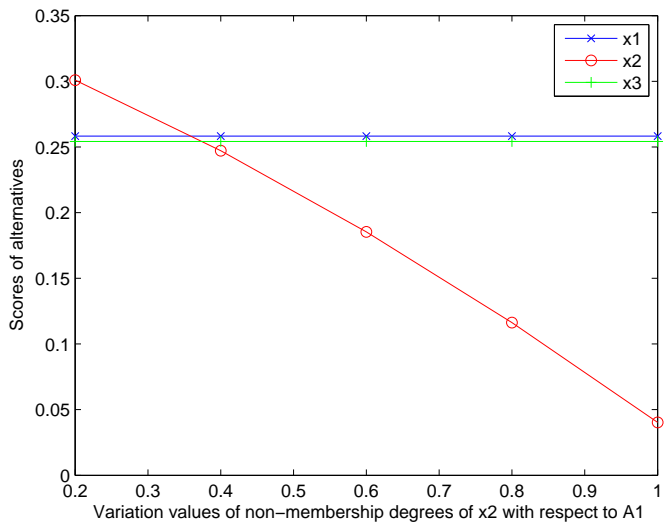

Figure 2: Ranking order sensitivity to the non-membership degrees of $x_{2}$ with respect to the first attribute $A_{1}$ by our Method I 


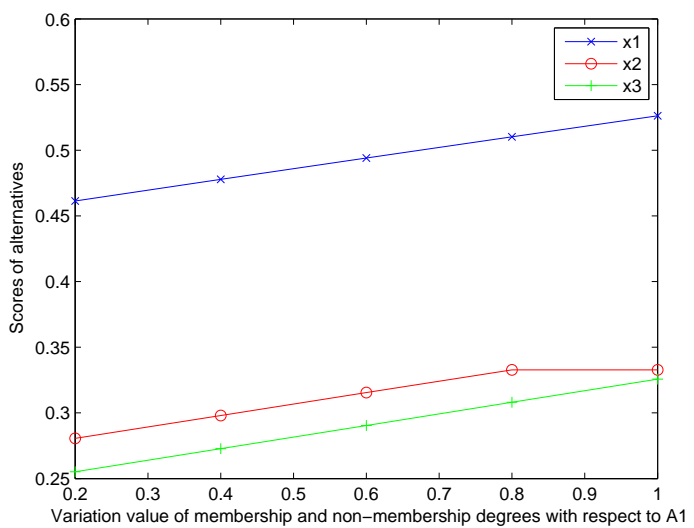

Figure 3: Ranking order sensitivity to the membership and non-membership degrees with respect to the first attribute $A_{1}$

$[0,1]$. Therefore, we can determine the step size $\Delta_{i j, t_{k}}$ according to the condition $\mu_{i j, t_{k}}+\Delta_{i j, t_{k}}, v_{i j, t_{k}}-$ $\Delta_{i j, t_{k}} \in[0,1]$.

Now, we take Example 5 (Section 5.1) as an example, we can obtain the preference order of the alternatives by changing the membership and non-membership degrees of three attributes, the details are shown in Figures 3-5, which also show the desirable alternatives will remain constant when the variation values of the membership and non-membership degrees with respect to the three attributes vary in the range from 0.1 to 1 . But regarding the range of membership degree and non-membership degree, the ranking order of the two alternatives $A_{2}$ and $A_{3}$ will change with the membership and non-membership degrees. It demonstrates that the alternatives $A_{2}$ and $A_{3}$ are more sensitive to membership and non-membership degrees than $A_{1}$.

\section{Conclusions}

In this paper, we have proposed two kinds of dynamic fuzzy multi-attribute decision making (DIF-MADM) methods in order to overcome the drawback of the existing DIF-MADM methods: the first one is using the ER methodology; the other one is based on the modified dynamic intuitionistic fuzzy weighted geometric aggregation (MDIFWG) operator. From the experimental results of several examples shown in Tables 3, 5, 7 and the comparative analysis, we can concluded that the proposed methods can overcome the drawbacks of some existing DIF-MADM methods, the details are shown in Table 9, so have shown the good potential in handling DIFMADM problem.

\section{Acknowledgments}

This work is supported by National Natural Science Foundation of P. R. China (Grant no. 61673320); Chinese Scholarship Council of the Ministry of Education ([2016]5112); The Application Basic Research Plan Project of Sichuan Province (No.2015JY0120); The Scientific Research Project of Department of Education of Sichuan Province (15TD0027); Natural Science Foundation of Guangdong Province (2016A030310003). 


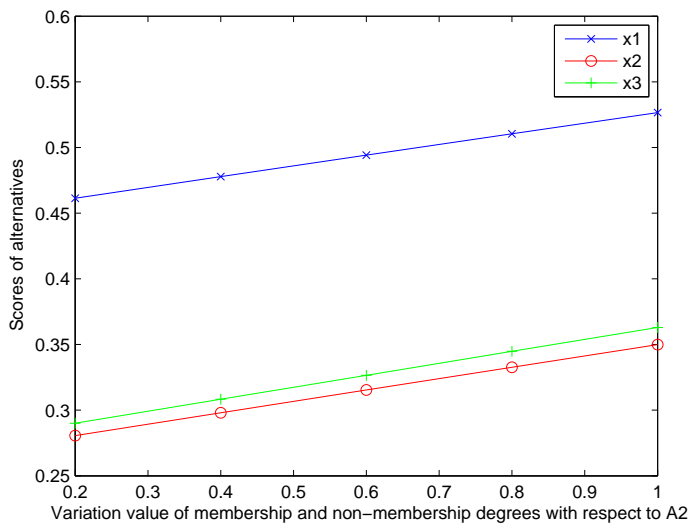

Figure 4: Ranking order sensitivity to the membership and non-membership degrees with respect to the second attribute $A_{2}$

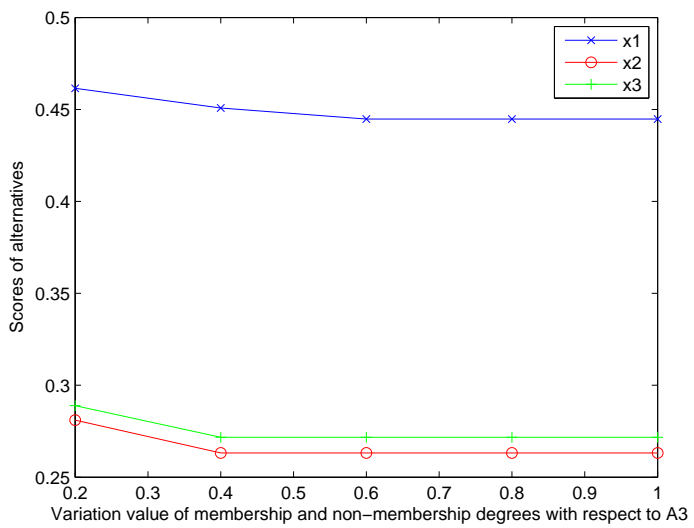

Figure 5: Ranking order sensitivity to the membership and non-membership degrees with respect to the third attribute $A_{3}$

Table 9: Corresponding Drawbacks and solutions by proposed methods

\begin{tabular}{ccc}
\hline & Method I & Method II \\
\hline Drawback A & Y & N/A \\
Drawback B & Y & Y \\
Drawback C & Y & Y \\
\hline
\end{tabular}




\section{References}

[1] K. T. Atanassov, Intuitionistic fuzzy sets, Fuzzy Sets Syst. 20 (1) (1986) 87-96.

500 [2] K. T. Atanassov, More on Intuitionistic fuzzy sets, Fuzzy Sets Syst. 33 (1) (1989) 37-46.

[3] K. T. Atanassov, New operators defined over the intuitionistic fuzzy sets, Fuzzy Sets Syst. 61 (2) (1994) 137-142.

[4] O. Bali, M. Dagdeviren, S. Gumus, An integrated dynamic intuitionistic fuzzy MADM approach for personnel promotion problem, Kybernetes, 44 (2015) 1422-1436.

[5] T. Bao, X. Xie, P. Long, Z. Wei, MADM method based on prospect theory and evidential reasoning approach with unknown attribute weights under intuitionistic fuzzy environment, Expert Systems with Applications, 88 (2017) 305-317.

[6] B. F. Baird, Managerial Decisions Under Uncertainty, an Introduction to the Analysis of Decision Making, Wiley, New York (1989).

[7] M. A. Butt, M. Akram, A new intuitionistic fuzzy rule-based decision-making system for an operating system process scheduler, Springerplus, 5 (2016) 1547.

[8] S. Cevik Onar, B. Oztaysi, C. Kahraman, Dynamic intuitionistic fuzzy multi-attribute aftersales performance evaluation, Complex and Intelligent Systems, (2017) DOI 10.1007/s40747-017-0047-7.

[9] S. Chen, J. Liu, H. Wang, J.C. Augusto, A group decision making model for partially ordered preference under uncertainty, Information Fusion, 25 (2015) 32-41.

515 [10] S. M. Chen, S. H. Cheng, C. H. Chiou, Fuzzy multiattribute group decision making based on intuitionistic fuzzy sets and evidential reasoning methodology, Information Fusion, 27 (2016) 215-227.

[11] S. M. Chen, C. H. Chang, Fuzzy multiattribute decision making based on transformation techniques of intuitionistic fuzzy values and intuitionistic fuzzy geometric averaging operators, Inf. Sci., 352-353 (2016) 133-149.

[12] Y. Chen, B. Li, Dynamic multi-attribute decision making model based on triangular intuitionistic fuzzy numbers, Sci Iran, 18 (2011) 268-274.

[13] A. P. Dempster, A generalization of Bayesian inference, J. Royal Stat. SOC., 30(1968)205-247.

[14] G. Shafer, A Mathematical Theory of Evidence Princeton, NJ: Princeton University Press, 1976.

[15] S. Gumus, O. Bali, Dynamic Aggregation Operators Based on Intuitionistic Fuzzy Tools and Einstein Operations, Fuzzy Information and Engineering, 9 (2017) 45-65.

[16] K. Guo, W. Li, An attitudinal-based method for constructing intuitionistic fuzzy information in hybrid MADM under uncertainty, Inf. Sci. 208 (2012) 28-38.

[17] J. Y. Huang, Intuitionistic fuzzy Hamacher aggregation operators and their application to multiple attribute decision making, J. Intell. Fuzzy Syst. 27 (1) (2014) 505-513.

[18] L. He, H. Teng, GRA model for dynamic hybrid multiple attribute decision making, Journal of Intelligent and Fuzzy Systems, 27 (2014) 1067-1075.

[19] G. Li, G. Kou, Y. Peng, Dynamic fuzzy multiple criteria decision making for performance evaluation, Technol Econ Dev Eco, 21 (2015) 705-719.

[20] R. Lin, X. Zhao, G. Wei, Fuzzy number intuitionistic fuzzy prioritized operators and their application to multiple attribute decision making, J. Intell. Fuzzy Syst. 24 (4) (2013) 879-888.

535 [21] A. F. Liu, TOPSIS method for multiple attribute decision making under trapezoidal intuitionistic fuzzy environment, J. Intell. Fuzzy Syst. 26 (5) (2014) 2403-2409.

[22] J. Liu, L. Martinez, Y. M. Wang, Extended Belief Rule Base Inference Methodology, Proceedings of 2008 3rd International Conference on Intelligent System and Knowledge Engineering, (2008) 1415-1450.

[23] J. Liu, J. B. Yang, D. Ruan, L. Martinez, J. Wang, Self-tuning of fuzzy belief rule bases for engineering system safety analysis, Ann Oper Res, 163 (2008) 143-168.

[24] J. Liu, J. B. Yang, J. Wang, H. S. Sii, Y. M. Wang, Fuzzy Rule-Based Evidential Reasoning Approach for Safety Analysis, Int J Gen Syst, 33 (2004) 183-204.

[25] S. Liu, T.A. Moughal, A Novel Method for Dynamic Multicriteria Decision Making with Hybrid Evaluation Information, J Appl Math, 2014 (2014) 1-11.

545 [26] J. H. Park, H. J. Cho, Y. C. Kwun, Extension of the VIKOR method to dynamic intuitionistic fuzzy multiple attribute decision making, Comput Math Appl, 65 (2013) 731-744.

[27] Z. X. Su, M. Y. Chen, G. P. Xia, L. Wang, An interactive method for dynamic intuitionistic fuzzy multi-attribute group decision making, Expert Systems with Applications, 38 (2011) 15286-15295.

[28] W. Wang, X. Liu, Intuitionistic fuzzy geometric aggregation operators based on Einstein operations, Int. J. Intell. Syst. 26 (11) (2011) 1049-1075.

[29] J. Q. Wang, J. J. Li, Multi-criteria fuzzy decision-making method based on cross entropy and score functions, Expert Systems with Applications, 38 (1) (2011) 1032-1038.

[30] J. Q. Wang, H. Y. Zhang, Multicriteria decision-making approach based on Atanassov?s intuitionistic fuzzy sets with incomplete certain information on weights, IEEE Trans. Fuzzy Syst. 21(3) (2013) 510-515. 
[31] G. W. Wei, X. Zhao, An approach to multiple attribute decision making with combined weight information in interval-valued intuitionistic fuzzy environment, Control Cybern, 41 (2012) 97-112.

[32] G. W. Wei, Some geometric aggregation functions and their application to dynamic multiple attribute decision making in the intuitionist fuzzy setting, International Journal of Uncertainty, Fuzziness and Knowledge-Based Systems, 17 (2009) 179-196.

[33] Y. Xu, Y. Wang, X. Miu, Multi-attribute decision making method for air target threat evaluation based on intuitionistic fuzzy sets, Syst. Eng. Electr. 23 (6) (2012) 891-897.

[34] Z. S. Xu, Intuitionistic fuzzy aggregation operators, IEEE Trans. Fuzzy Syst. 15 (6) (2007) 1179-1187.

[35] Z. S. Xu, Choquet integrals of weighted intuitionistic fuzzy information, Inf. Sci. 180 (5) (2010) 726-736

[36] Z. S. Xu, Intuitionistic fuzzy multiattribute decision making: An interactive method, IEEE Trans. Fuzzy Syst. 20 (3) (2012) 514-525.

[37] Z. S. Xu, Approaches to Multi-Stage Multi-Attribute Group Decision Making, International Journal of Information Technology and Decision Making, 10 (2011) 121-146.

[38] Z. S. Xu, R.R. Yager, Some geometric aggregation operators based on intuitionistic fuzzy sets, Int J Gen Syst, 35 (2006) 417-433.

570 [39] Z. S. Xu, R.R. Yager, Dynamic intuitionistic fuzzy multi-attribute decision making, International Journal of Approximate Reasoning, 48 (2008) 246-262.

[40] Z. S. Xu, Q.L. Da, An overview of operators for aggregating information, International Journal of Intelligent Systems, 18 (2003) 953-969.

[41] Z. S. Xu, A method based on the dynamic weighted geometric aggregation operator for dynamic hybrid multiattribute group decision making, International Journal of Uncertainty, Fuzziness and Knowledge-Based Systems, 17 (2009) 15 ?a33.

[42] P. Yu, J. Zhang, An algorithmic method to extend TOPSIS for multiple attribute decision making under intuitionistic fuzzy setting, J. Intell. Fuzzy Syst. 26 (5) (2014) 2315-2322.

[43] H. Zhao, Z.S. Xu, M.F. Ni, S. Liu, Generalized aggregation operators for intuitionistic fuzzy sets, Int. J. Intell. Syst. 25 (1) (2010) 1-30.

[44] S. Zhang, D. Yu, Some geometric Choquet aggregation operators using Einstein operations under intuitionistic fuzzy environment, J. Intell. Fuzzy Syst. 26 (1) (2014) 491-500.

[45] S. Zeng, T. Balezentis, J. Chen, G. Luo, A Projection Method for Multiple Attribute Group Decision Making with Intuitionistic Fuzzy Information, Informatica-Lithuan, 24 (2013) 485-503.

[46] S. Zhou, W. Chang, Approach to multiple attribute decision making based on the Hamacher operation with fuzzy number intuitionistic fuzzy information and their application, J. Intell. Fuzzy Syst. 27 (3) (2014) 1087-1094.

[47] J. B. Yang, Rule and utility based evidential reasoning approach for multiattribute decision analysis under uncertainty, Eur. J. Oper. Res. 131 (1) (2001) 31-61.

[48] J. B. Yang, D.L. Xu, On the evidential reasoning algorithm for multiple attribute decision analysis under uncertainty, IEEE Trans. Syst. Man Cybernet. - Part A: Syst. Humans 32 (3) (2002) 289-304.

[49] J. B. Yang, J. Liu, J. Wang, H. S. Sii, H. W. Wang, Belief rule-base inference methodology using the evidential reasoning Approach-RIMER, IEEE Transactions on Systems, Man, and Cybernetics - Part A: Systems and Humans, 36 (2006) 266-285.

[50] J. B. Yang, M. G. Singh, An evidential reasoning approach for multiple-attribute decision making with uncertainty, IEEE Trans. Syst. Man Cybernet. - Part A: Syst. Humans 24 (1) (1994) 1-18.

[51] J. B. Yang, Y. M. Wang, D. L. Xu, K. S. Chin, The evidential reasoning approach for MADA under both probabilistic and fuzzy uncertainties, Eur. J. Oper. Res. 171 (1) (2006) 309-343.

[52] Z. Yang, J. Li, L. Huang, Y. Shi, Developing dynamic intuitionistic normal fuzzy aggregation operators for multiattribute decision-making with time sequence preference, Expert Systems with Applications, 82 (2017) 344-356.

600 [53] H. Zhu, J. Zhao, Y. Xu, L. Du, Interval-Valued Belief Rule Inference Methodology Based on Evidential ReasoningIRIMER, International Journal of Information Technology and Decision Making, 15 (2016) 1345-1366. 